Supporting Information

\title{
Application of Life Cycle Assessment and Machine Learning for High-Throughput Screening of Green Chemical Substitutes
}

Xinzhe Zhu ${ }^{\dagger}$, Chi-Hung $\mathrm{Ho}^{\dagger}$, Xiaonan Wang*

Department of Chemical and Biomolecular Engineering, National University of

Singapore,

4 Engineering Drive 4, 117585 Singapore

$\dagger$ These authors contribute equally to this work.

* Corresponding author:

Tel: +65 66016221

Email: chewxia@nus.edu.sg

Summary Information

Number of Pages: 27

Number of Figures: 6 
Number of Tables: 6 


\section{METHOLOGY SECTION}

\section{Principal component analysis (PCA) method}

The 125 molecular descriptors from Rdkit may contain data noises and meanwhile increase the computation time. PCA is a well-known multivariate analysis method, which could detect the internal relationships among variables and reduce the dimensions of molecular descriptors with an orthogonal transformation to convert a set of possible correlated variables into a set of linearly uncorrelated variables (i.e. principal components). The first principal component retains the largest variables with linear correlations and the second one provides the best linear combination of residual variables. The succeeding principal components are acquired with the same way until all the original data is exhausted ${ }^{1}$. The conceptual diagram of data structures before and after PCA is shown in Fig. S3. In this study, python code from Scikit-learn was used to conduct principal component analysis. 


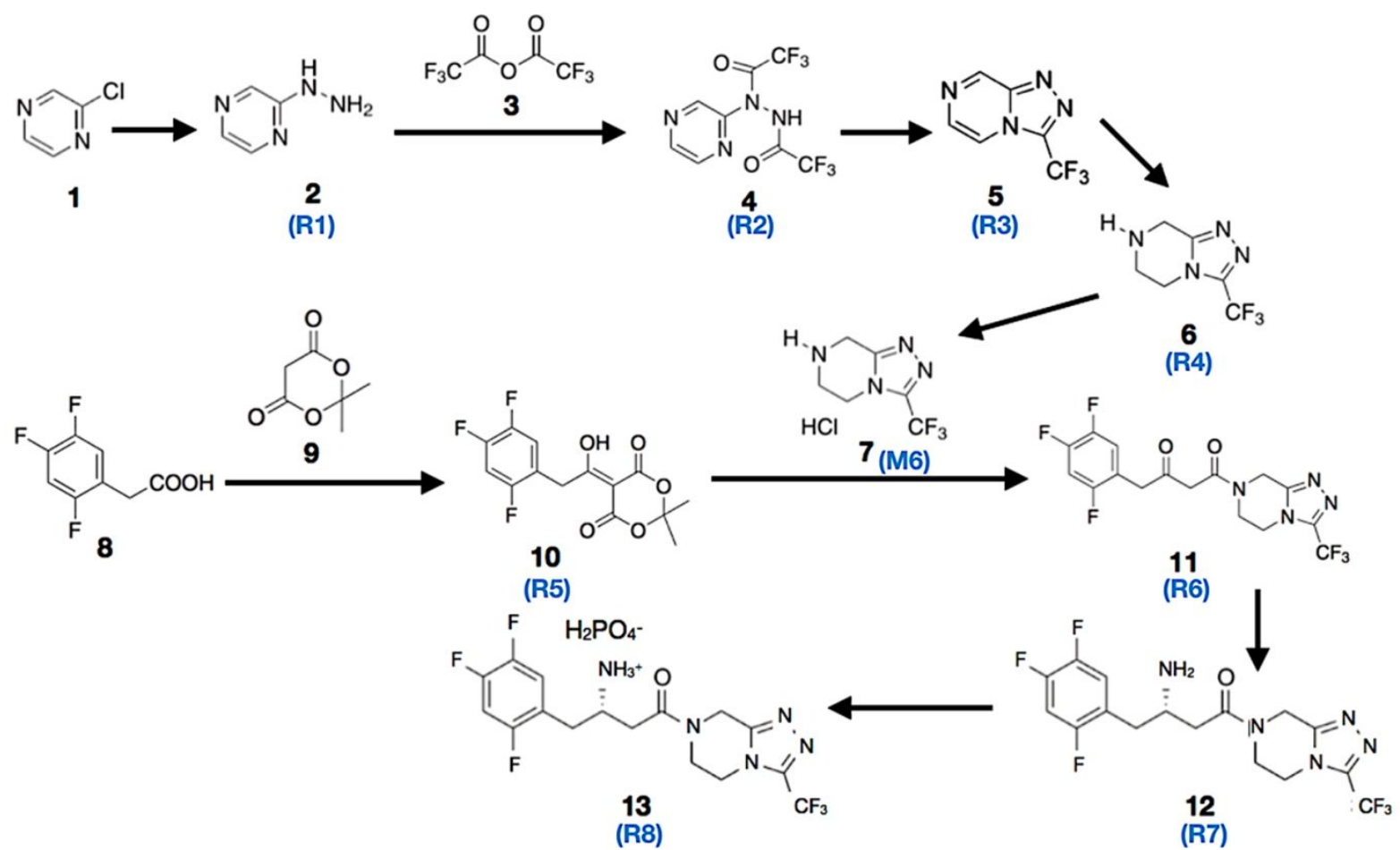

Fig. S1 Synthetic route of sitagliptin production used in the study ${ }^{2}$ 


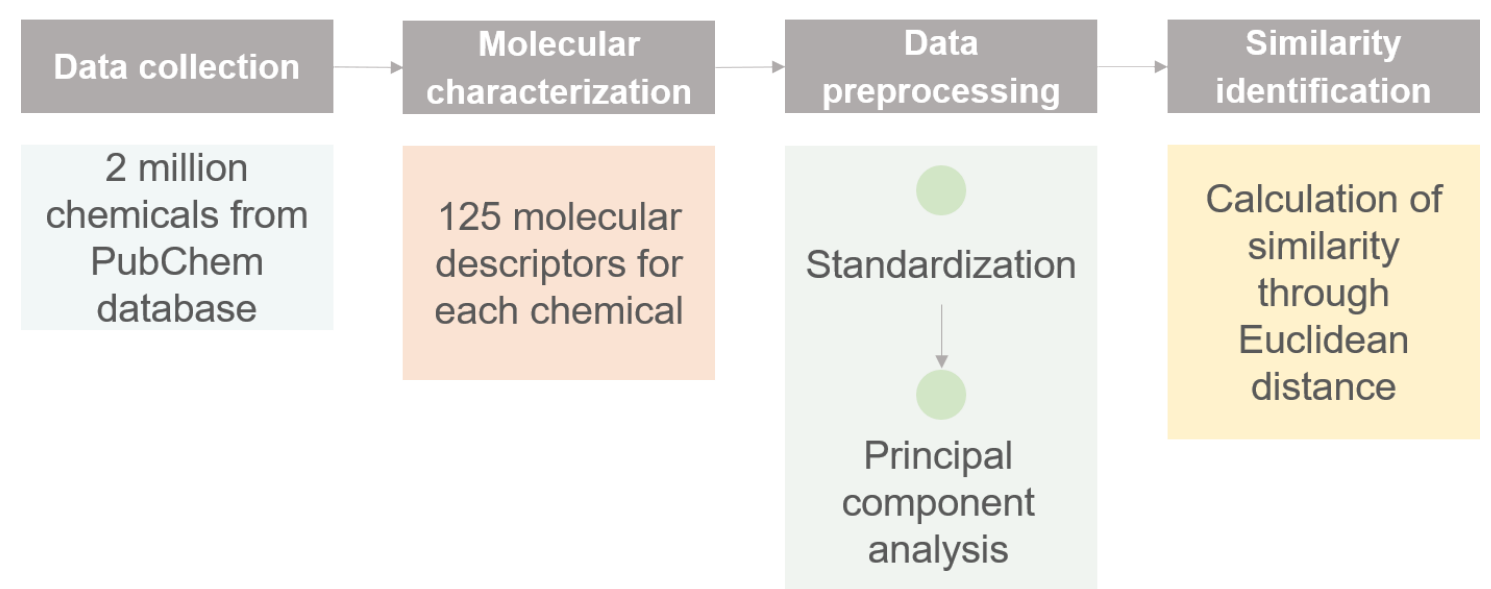

Fig. S2 Flowsheet of the identification of similar chemicals from PubChem database

\begin{tabular}{|c|c|c|c|c|c|c|c|c|c|c|c|}
\hline & Descriptor 1 & 2 & $\cdots$ & 124 & 125 & & $\begin{array}{l}\text { Principle } \\
\text { component } 1\end{array}$ & 2 & $\ldots$ & $n-1$ & $\mathrm{n}$ \\
\hline Chemical 1 & Numeric value & $\ldots$ & $\ldots$ & $\ldots$ & $\ldots$ & Chemical 1 & Numeric value & $\ldots$ & $\ldots$ & $\ldots$ & $\ldots$ \\
\hline 2 & $\ldots$ & $\ldots$ & $\ldots$ & $\ldots$ & $\ldots$ & 2 & $\ldots$ & $\ldots$ & $\ldots$ & $\ldots$ & $\ldots$ \\
\hline . & $\ldots$ & $\ldots$ & $\ldots$ & $\ldots$ & $\ldots$ & . & $\ldots$ & $\ldots$ & $\ldots$ & $\ldots$ & $\ldots$ \\
\hline$\cdot$ & & & & & & . & \multicolumn{5}{|c|}{ Uncorrelated variables } \\
\hline $1,999,999$ & $\ldots$ & $\ldots$ & $\ldots$ & $\ldots$ & $\ldots$ & $1,999,999$ & $\ldots$ & ... & $\ldots$ & ... & $\ldots$ \\
\hline $2,000,000$ & $\ldots$ & $\ldots$ & $\ldots$ & $\ldots$ & $\ldots$ & $2,000,000$ & $\ldots$ & $\ldots$ & $\ldots$ & $\ldots$ & $\ldots$ \\
\hline
\end{tabular}

Fig. S3 Data structures before and after PCA 


\section{Selection of number of principle components}

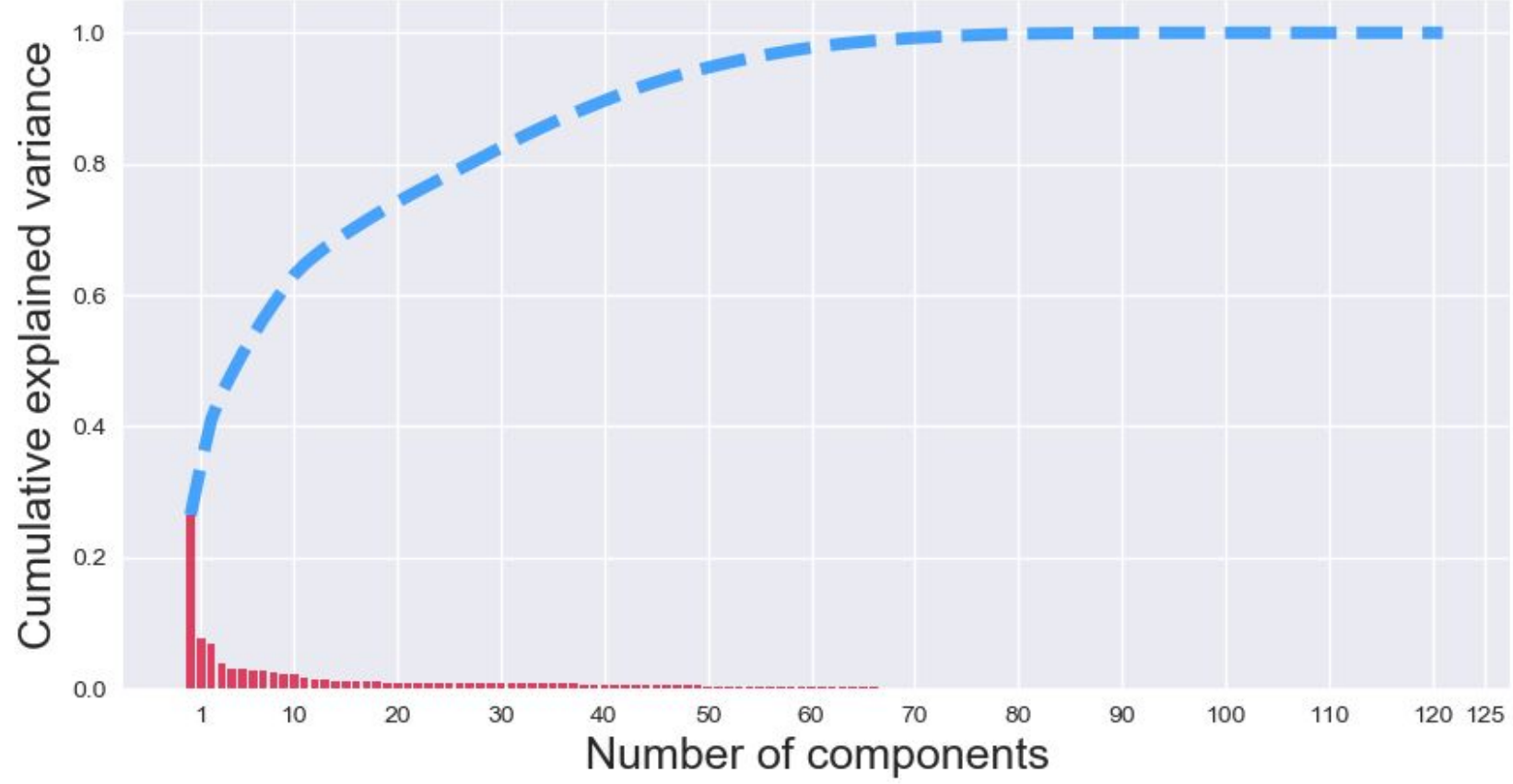

Fig. S4 Number of descriptors extracted by PCA against the cumulative variance preserved by the corresponding descriptors. The red referred to the information preserved of each principle component, while the blue one was the cumulative values of preserved principle components. 

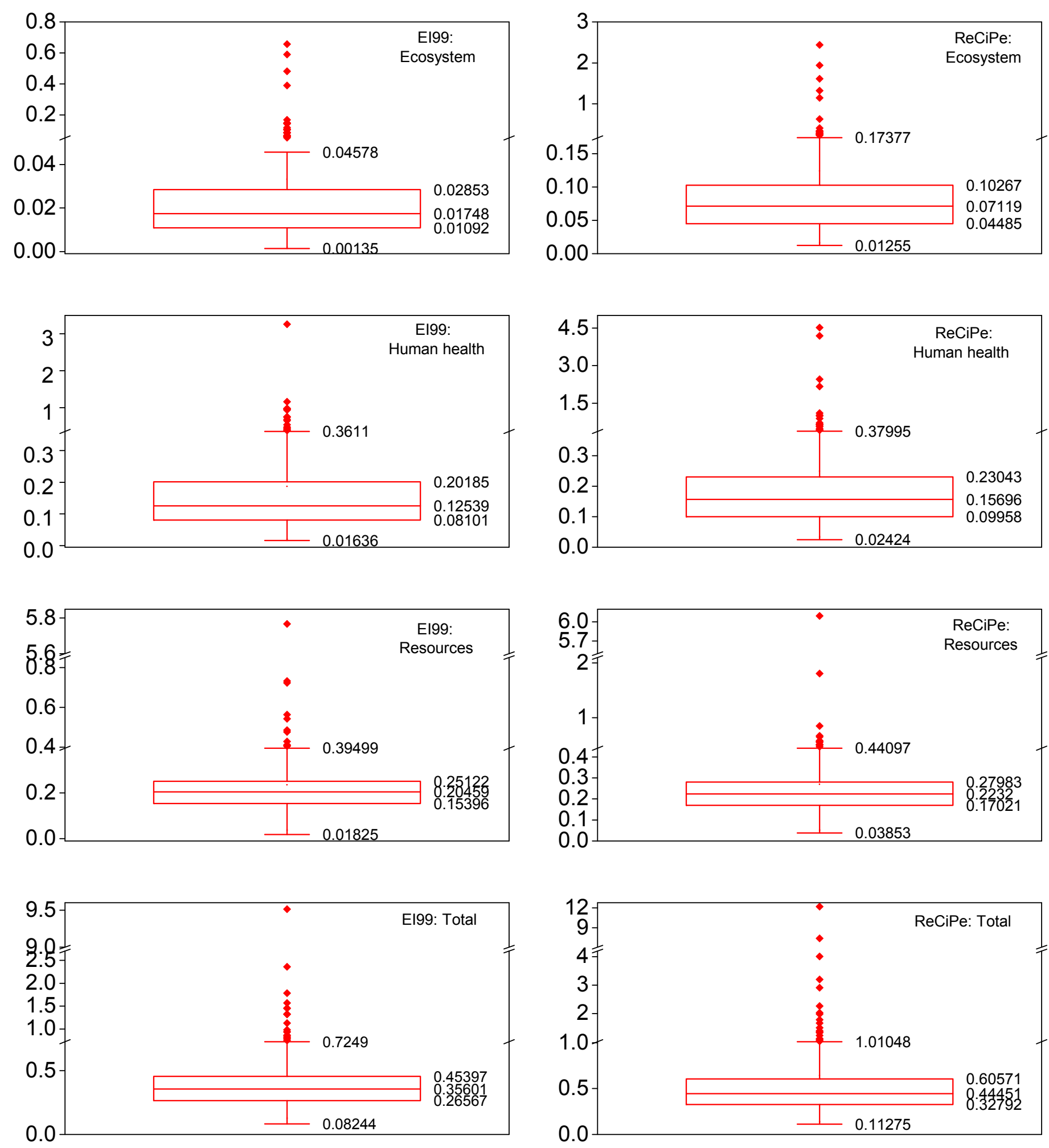

Fig. S5 LCA data distributions of nonionic organic chemicals from Ecoinvent v3.5 database 

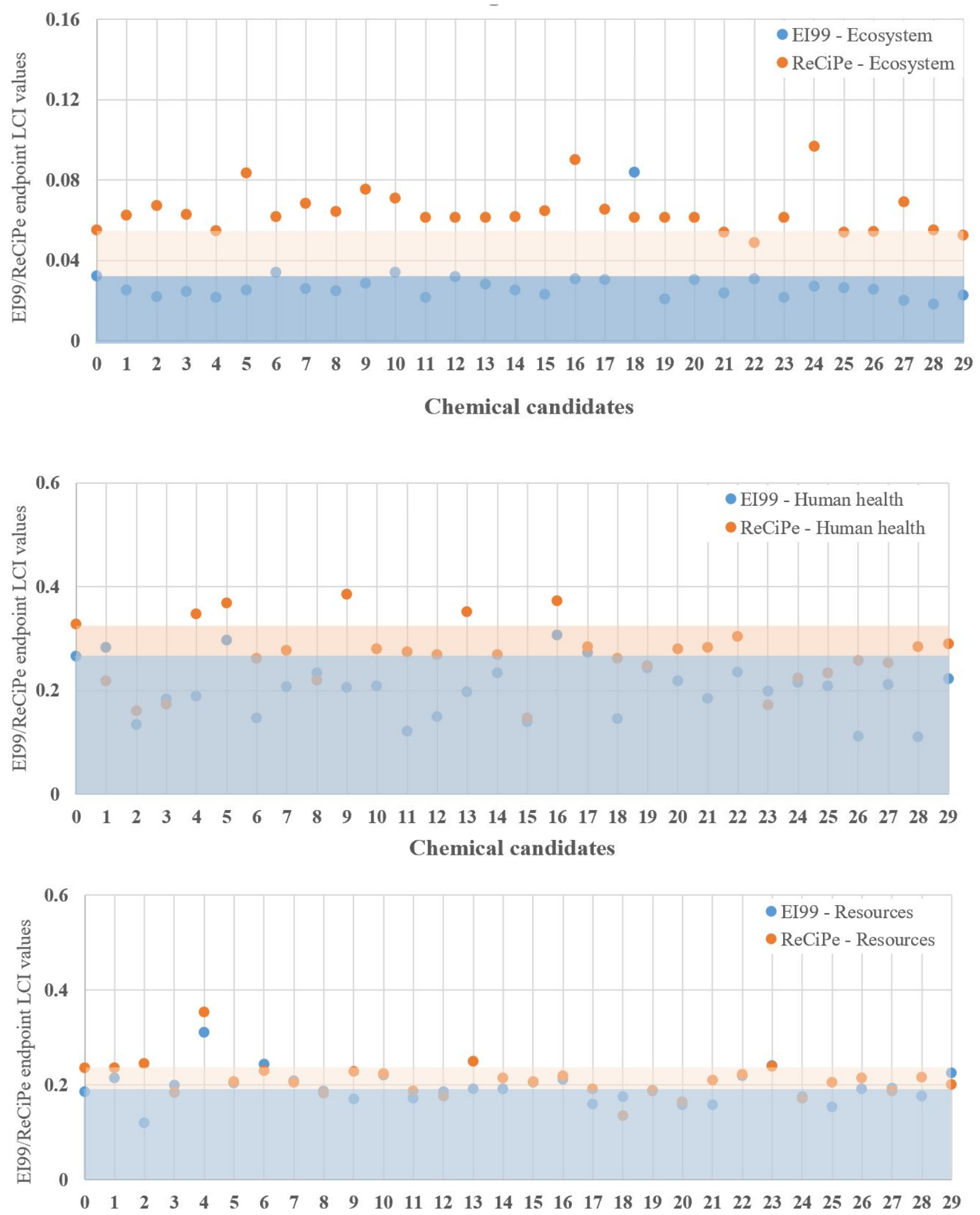

Chemical candidates

Fig. S6 LCIA prediction values of 30 most similar chemicals with trifluoroacetic anhydride using individual categories of EI99 and ReCiPe methods. The blue/pink areas referred to the ranges containing the chemicals that had lower individual EI99/ReCiPe values than that of target chemical. 
Table $\mathbf{S 1}$ Mass balance throughout the sitagliptin manufacturing process ${ }^{2}$

\begin{tabular}{|c|c|c|c|c|c|c|c|c|c|c|c|c|c|c|c|c|c|c|c|c|c|c|}
\hline Mass flow rate (kg/hr) & 1 & 2 & 3 & 4 & 5 & 6 & 7 & 8 & 9 & 10 & 11 & 12 & 13 & 14 & 15 & 16 & 17 & $\begin{array}{c}18 \\
\text { (solid) }\end{array}$ & $\begin{array}{c}18 \\
\text { (solution) }\end{array}$ & 19 & 20 & 21 \\
\hline 1 & 0.03409 & 0.00000 & 03409 & 00341 & .00000 & .00000 & 0.00341 & 00068 & 00273 & 00000 & 0.00273 & 00273 & .00000 & 0.00273 & 0.00273 & 0.00000 & 00000 & .00000 & 0.00273 & 0.00000 & 0.00273 & 0.000 \\
\hline $\mathrm{H}_{2} \mathrm{O}$ & 0.00000 & 2657 & 02657 & 0.02657 & 10000 & 00000 & 0.02657 & 2657 & 10000 & .00000 & 1.00000 & 0.00000 & 0.00000 & 0.00000 & 0.00000 & 0.00000 & .00000 & 00000 & 0.00000 & 0.00000 & .00000 & 0.00000 \\
\hline $\mathrm{NH}_{2} \mathrm{NH}_{2}$ & 0.00000 & 01431 & 0.01431 & 0.00572 & 0.00000 & 0.00000 & 0.00572 & 0.00572 & 100000 & 0.00000 & 0.00000 & 0.00000 & 0.00000 & 0.00000 & 0.00000 & 0.00000 & 0.00000 & 0.00000 & 0.00000 & 0.00000 & 0.00000 & 0.00000 \\
\hline 2 & 0.00000 & 0.00000 & 0.00000 & 2950 & 00000 & 0. & 0.02950 & 61 & 0.025 & 0.000 & 0.02589 & 0.02589 & 0.00000 & 0.02589 & 0.00777 & 0.00000 & 0.00000 & 0.00000 & 0.00777 & 0.00000 & 0.00777 & 0.00000 \\
\hline $\mathrm{HCl}$ & 00000 & 10000 & 10000 & 09977 & 00000 & 0000 & 00977 & 00977 & 00000 & 00000 & 0.00000 & 0.00000 & 0.00000 & 0.00000 & 0.00000 & 00000 & 00000 & 00000 & 0.00000 & 0.000000 & 0.00000 & 0.00000 \\
\hline 2-propanol & 0.00000 & 0.00000 & 0.00000 & 0.00000 & 00147 & 0.02950 & 0.02950 & 00000 & 02950 & 0.02802 & 0.00147 & 0.01353 & 0.00000 & 0.01353 & 0.01353 & 0.00000 & 0.00000 & 0.00000 & 0.01353 & 0.000 & 0.01353 & 0.00000 \\
\hline dichlorometh & 0.00000 & 0.00000 & 0.00000 & 0.00000 & 0.01327 & 0.26548 & 0.26548 & 0.00000 & 0.26548 & 0.25220 & 0.01327 & 0.01327 & 0.00000 & 0.01327 & 0.01327 & 0.00000 & 0.00000 & 0.00000 & 0.01327 & 0.00000 & 0.01327 & 0.00000 \\
\hline IPAC & 0.00000 & 0.00000 & 0.00000 & 0.00000 & 0.00000 & 0.00000 & 0.00000 & 0.00000 & 0.00000 & 0.00000 & 0.00000 & 0.10000 & 0.00000 & 0.10000 & 0.10000 & 0.00000 & 0.00000 & 0.00000 & 0.10000 & 0.00000 & 0.10000 & 0.00000 \\
\hline 3 & 0.00000 & 00000 & 00000 & 0.00000 & 0.00000 & 00000 & 0.00000 & 0.00000 & 0.00000 & 0.00000 & 0.00000 & 0.00000 & 0.09877 & 0.09877 & 0.02963 & 0.00000 & 0.00000 & .00000 & 0.02963 & 0.00000 & 0.02963 & 0.00000 \\
\hline 4 & 10000 & & & 0.00000 & & & 00000 & 0.00000 & 00000 & & 0.00000 & 0.00000 & & 0.00000 & 0.04973 & & & 03481 & & & & 0000 \\
\hline Trifluoroaceti & 0.00000 & 0.00000 & 00000 & 0.00000 & 0.00000 & 00000 & 0.00000 & 0,00000 & 0.00000 & 0.00000 & 0.00000 & 0.00000 & 0.00000 & 0.00000 & 0.03753 & 0.00000 & 0.00000 & 0.00000 & 0.03753 & 0.00000 & 0.03753 & 0.00000 \\
\hline heptane & 0.00000 & 00000 & 00000 & 0.00000 & 0.00000 & 0.00000 & 0.00000 & 0.00000 & 0.00000 & 0.00000 & 0.00000 & 0.00000 & 0.00000 & 0.00000 & 0.00000 & 0.10000 & 0.00500 & .00000 & 0.00000 & 0.00000 & 0.00000 & 0.09500 \\
\hline Sup erphospho & 0.00000 & 0.00000 & 0.00000 & 0.00000 & 0.00000 & 0.00000 & 0.00000 & 0.00000 & 0.00000 & 0.00000 & 0.00000 & 0.00000 & 0.00000 & 0.00000 & 0.00000 & 0.00000 & 0.00000 & 0.00000 & 0.00000 & 0.01000 & 0.01000 & 0.00000 \\
\hline 5 & 0.00000 & 0.00000 & 0.00000 & 0.00000 & 0.00000 & 00000 & 0.00000 & 0.00000 & 0.00000 & 0.00000 & 0.00000 & 0.00000 & 0.00000 & 0.00000 & 0.00000 & 0.00000 & 0.00000 & 0.00000 & 0.00000 & 0.00000 & 0.00000 & 0.00000 \\
\hline $\mathrm{H}_{2}$ gas & 0.00000 & 0.00000 & .00000 & 0.00000 & 0.00000 & 0.00000 & 0.00000 & 0.00000 & 0.00000 & 0.00000 & 0.00000 & 0.00000 & 0.00000 & 0.00000 & 0.00000 & 0.00000 & 0.00000 & 1.00000 & 0.00000 & 0.00000 & 0.00000 & 0.00000 \\
\hline 6 & & & & 0.00000 & 00000 & 10000 & 0.00000 & 0.00000 & 0.00000 & & 0.000 & 0.00000 & & 0.000 & 0.000 & & & 001 & & & & \\
\hline 7 & 0.00000 & 0.00000 & 0.00000 & 0.00000 & 0.00000 & 0.00000 & 0.00000 & 0.00000 & 0.00000 & 0.00000 & 0.00000 & 0.00000 & 0.00000 & 0.00000 & 0.00000 & 0.00000 & 0.00000 & 0.00000 & 0.00000 & 0.00000 & 0.00000 & 0.00000 \\
\hline IPA & 0.00000 & 0.00000 & 0.00000 & 0.00000 & 0.00000 & 0.00000 & 0.00000 & 0.00000 & 0.00000 & 0.00000 & 0.00000 & 0.00000 & 0.00000 & 0.00000 & 0.00000 & 0.00000 & 0.00000 & 0.00000 & 0.00000 & 0.00000 & 0.00000 & 0.00000 \\
\hline 8 & 0.00000 & 0.00000 & 0.00000 & 0.00000 & 0.00000 & 0.00000 & 0.00000 & 0.00000 & 0.00000 & 0.00000 & 0.00000 & 0.00000 & 0.00000 & 0.00000 & 0.00000 & 0.00000 & 0.00000 & 0.00000 & 0.00000 & 0.00000 & 0.00000 & 0.00000 \\
\hline 9 & & & & & & & 00 & & & & & & & & & & & & & & & \\
\hline PivCl & 0.00000 & 0.00000 & 0.00000 & 0.00000 & 0.00000 & 0.00000 & 0.00000 & 0.00000 & 0.00000 & 0.00000 & 0.00000 & 0.00000 & 0.00000 & 0.00000 & 0.00000 & 0.00000 & 0.00000 & .00000 & 0.00000 & 0.00000 & 0.00000 & 0.00000 \\
\hline IPEA & 0.00000 & 0.00000 & 0.00000 & 0.00000 & 0.00000 & 0.00000 & 0.00000 & 0.00000 & 0.00000 & 0.00000 & 0.00000 & 0.00000 & 0.00000 & 0.00000 & 0.00000 & 0.00000 & 0.00000 & 0.00000 & 0.00000 & 0.00000 & 0.00000 & 0.00000 \\
\hline 10 & 0.00000 & 0.00000 & 0.00000 & 0.00000 & 0.00000 & 0.00000 & 0.00000 & 0.00000 & 0.00000 & 0.00000 & 0.00000 & 0.00000 & 0.00000 & 0.00000 & 0.00000 & 0.00000 & 0.00000 & 0.00000 & 0.00000 & 0.00000 & 0.00000 & 0.00000 \\
\hline by-product fre & 0.00000 & 0.00000 & 00000 & 0,00000 & 0.00000 & 0.00000 & 0.00000 & 0.00000 & 0.00000 & 0.00000 & 0.00000 & 0.00000 & 0.00000 & 0.00000 & 0.00000 & 00000 & 0.00000 & .00000 & 0.00000 & 0.00000 & 0.00000 & 0.00000 \\
\hline TFA & 0.00000 & 0.00000 & 00000 & 0.00000 & 0.00000 & 0.00000 & 0.00000 & 0.00000 & 0.00000 & 0.00000 & 0.00000 & 0.00000 & 0.00000 & 0.00000 & 0.00000 & 0.00000 & 0.00000 & 0.00000 & 0.00000 & 0.00000 & 0.00000 & 0.00000 \\
\hline 11 & 0.00000 & 0.00000 & 0.00000 & 0.00000 & 0.00000 & 0.00000 & 0.00000 & 0.00000 & 0.00000 & 0.00 & 0.00000 & 0.00000 & & 0.00000 & 0.00000 & & 00 & .00000 & 1000 & 0.00000 & 0.00000 & 0.00000 \\
\hline by-product & 0.00000 & 0,00000 & 0.00 & 0.00000 & 0.00000 & 0000 & 0.00000 & 0.00000 & 0.00000 & 0.00000 & 0.00000 & 0.00000 & 0.00000 & 0.00000 & 0.00000 & 0.00000 & 0.00000 & 0.00000 & 0.00000 & 0.00000 & 0.00000 & 0.00000 \\
\hline & 0.00000 & . & & 0.00000 & 0.00000 & 0.0000 & 0.00000 & 0.00000 & 0.00000 & 0.000 & 0.00000 & 0.00000 & & 0.00000 & 0.00000 & 00 & 0.00000 & 0.00000 & 0.00000 & 0.00000 & 0.00000 & 0.00000 \\
\hline & & & & & & & & & & & & & & & & & & & & & & 0000 \\
\hline 12 & 0000 & 0000 & 0000 & 0.00000 & 0.00000 & 0.00000 & 0.00000 & 0.00000 & 0.00000 & 0.00000 & 0.00000 & 0.00000 & 10000 & 0.00000 & 0.00000 & 0.00000 & 0.00000 & .00000 & 0.00000 & 0.00000 & 0.00000 & 0.00000 \\
\hline Acetone & 0.00000 & 0.00000 & 0.00000 & 0.00000 & 0.00000 & 0.00000 & 0.00000 & 0.00000 & 0.00000 & 0.00000 & 0.00000 & 0.00000 & 0.00000 & 0.00000 & 0.00000 & 0.00000 & 0.00000 & 0.00000 & 0.00000 & 0.00000 & 0.00000 & 0.00000 \\
\hline by-product from $\mathbf{R} 7$ & 0.00000 & 0.00000 & 0.00000 & 0.00000 & 0.00000 & 0.00000 & 0.00000 & 0.00000 & 0.00000 & 0.00000 & 0.00000 & 0.00000 & 0.00000 & 0.00000 & 0.00000 & 0.00000 & 0.00000 & 0.00000 & 0.00000 & 0.00000 & 0.00000 & 0.00000 \\
\hline $\mathrm{NaOH}$ & 0.00000 & 0.00000 & 0.00000 & 0.00000 & 0.00000 & 0.00000 & 0.00000 & 0.00000 & 0.00000 & 0.00000 & 0.00000 & 0.00000 & 0.00000 & 0.00000 & 0.00000 & 0.00000 & 0.00000 & 0.00000 & 0.00000 & 0.00000 & 0.00000 & 0.00000 \\
\hline $\mathrm{NaC}$ & 0000 & 10000 & & 0.00000 & 0.00000 & 0000 & 0.00000 & 0.00000 & & & & & & 10000 & 0.00000 & 00000 & 1.00000 & 00000 & 0.00000 & 0.00000 & 0.00000 & 0.00000 \\
\hline & 1000 & & & & & & & & & & & & & & & & & & & & 000 & 0000 \\
\hline & & & & & & & & & & & & & & & & & & & & & & \\
\hline pho & & & & 0000 & & 1000 & 0.00000 & 0000 & & & & & & 0000 & 00 & & & 00000 & & 0.00000 & 0.00000 & 00000 \\
\hline & 1000 & 1000 & 1000 & 0000 & 00000 & 0.00000 & 0000 & 0.00000 & 0.00000 & 0.00000 & 0.00000 & 0.00000 & 0.00000 & 0.00000 & 0.00000 & 0.00000 & 0.00000 & 0.00000 & 0.00000 & 0.00000 & 0.00000 & 0.00000 \\
\hline
\end{tabular}




\begin{tabular}{|c|c|c|c|c|c|c|c|c|c|c|c|c|c|c|c|c|c|c|c|c|c|c|}
\hline Mass flow rate (kg/hr) & 22 & 23 & 24 & 25 & 26 & 27 & 28 & 29 & 30 & 31 & 32 & 33 & 34 & 35 & 36 & $\begin{array}{c}37 \\
\text { (Pd } \\
\text { catalyst) }\end{array}$ & 38 & 39 & 40 & 41 & 42 & 43 \\
\hline 1 & 0.00000 & 0.00000 & 0.00000 & 0.00000 & 0.00000 & 0.00273 & 0.00000 & 0.00000 & 0.00000 & 0.00000 & 0.00000 & 0.00000 & 0.00000 & 0.00000 & 0.00000 & 0.00000 & 0.00000 & 0.00000 & 0.00000 & 0.00000 & 0.00000 & 0.00000 \\
\hline $\mathrm{H}_{2} \mathrm{O}$ & 0.00000 & 0.00000 & 0.00000 & 0.00000 & 0.00000 & 0.00000 & 0.00000 & 0.00000 & 0.00000 & 0.00000 & 0.00000 & 0.00000 & 0.00000 & 0.00000 & 0.00000 & 0.00000 & 0.00000 & 0.22857 & 0.00000 & 0.00000 & 0.00000 & 0.22857 \\
\hline $\mathrm{NH}_{2} \mathrm{NH}_{2}$ & 0.00000 & 0.00000 & 0.00000 & 0.00000 & 0.00000 & 0.00000 & 0.00000 & 0.00000 & 0.00000 & 0.00000 & 0.00000 & 0.00000 & 0.00000 & 0.00000 & 0.00000 & 0.00000 & 0.00000 & 0.00000 & 0.00000 & 0.00000 & 0.00000 & 0.00000 \\
\hline 2 & 0.00000 & 0.00000 & 0.00000 & 0.00000 & 0.00000 & 0.00777 & 0.00000 & 0.00000 & 0,00000 & 0.00000 & 0.00000 & 0,00000 & 0.00000 & 0.00000 & 0.00000 & 0.00000 & 0.00000 & 0.00000 & 0.00000 & 0.00000 & 0.00000 & 0.00000 \\
\hline $\mathrm{HCl}$ & 0.00000 & 0.00000 & 0.00000 & 0.00000 & 0.00000 & 0.00000 & 0.00000 & 0.00000 & 0.00000 & 0.00000 & 0.00000 & 0.00000 & 0.00000 & 0.00000 & 0.00000 & 0.00000 & 0.00000 & 0.09698 & 0.00000 & 0.00000 & 0.00000 & 0.10000 \\
\hline 2-propanol & 0.01218 & 0.00012 & 0.01206 & 0.00000 & 0.01206 & 0.00135 & 0.00000 & 0.00000 & 0.00000 & 0.00000 & 0.00000 & 0.00000 & 0.00000 & 0.00000 & 0.00000 & 0.00000 & 0.00000 & 0.00000 & 0.00000 & 0.00000 & 0.00000 & 0.00000 \\
\hline dichloromethane & 0.00000 & 0.00000 & 0.00000 & 0.00000 & 0.00000 & 0.01327 & 0.00000 & 0.00000 & 0.00000 & 0.00000 & 0.00000 & 0.00000 & 0.00000 & 0.00000 & 0.00000 & 0.00000 & 0.00000 & 0.00000 & 0.00000 & 0.00000 & 0.00000 & 0.00000 \\
\hline IPAC & 0.09000 & 0.00090 & 0.08910 & 0.01090 & 0.10000 & 0.01000 & 0.00000 & 0.00000 & 0.00000 & 0.00000 & 0.00000 & 0.00000 & 0.00000 & 0.00000 & 0.00000 & 0.00000 & 0.00000 & 0.00000 & 0.00000 & 0.00000 & 0.00000 & 0.00000 \\
\hline 3 & 0.00000 & 0.00000 & 0.00000 & 0.00000 & 0.00000 & 0.02963 & 0.00000 & 0.00000 & 0,00000 & 0.00000 & 0.00000 & 0.00000 & 0.00000 & 0.00000 & 0.00000 & 0.00000 & 0.00000 & 0.00000 & 0.00000 & 0.00000 & 0.00000 & 0.00000 \\
\hline 4 & 0.00000 & 0.00000 & 0.00000 & 0.00000 & 0.00000 & 0.01492 & 0.03481 & 0.00000 & 0.03481 & 0.00348 & 0.00348 & 0.00000 & 0.00000 & 0.00000 & 0.00348 & 0.00000 & 0.00348 & 0.00348 & 0.00000 & 0.00000 & 0.00000 & 0.00000 \\
\hline Trifluoroacetic acid & 0.00000 & 0.00000 & 0.00000 & 0.00000 & 0.00000 & 0.03753 & 0.00000 & 0.00000 & 0.00000 & 0.01182 & 0.01182 & 0.00000 & 0.00000 & 0.00000 & 0.01182 & 0.00000 & 0.01182 & 0.01182 & 0.00000 & 0.00000 & 0.00000 & 0.00000 \\
\hline heptane & 0.00000 & 0.00000 & 0.00000 & 0.00000 & 0.00000 & 0.00500 & 0.00000 & 0.00000 & 0.00000 & 0.00000 & 0.00000 & 0.00000 & 0.00000 & 0.00000 & 0.00000 & 0.00000 & 0.00000 & 0.00000 & 0.00000 & 0.00000 & 0.00000 & 0.00000 \\
\hline Sup erph osph oric acid & 0.00000 & 0.00000 & 0.00000 & 0.00000 & 0.00000 & 0.01000 & 0.00000 & 0.05000 & 0.05000 & 0.05000 & 0.05000 & 0.00000 & 0.00000 & 0.00000 & 0.05000 & 0.00000 & 0.05000 & 0.05000 & 0.00000 & 0.00000 & 0.00000 & 0.00000 \\
\hline 5 & 0.00000 & 0.00000 & 0.00000 & 0.00000 & 0.00000 & 0.00000 & 0.00000 & 0.00000 & 0.00000 & 0.01951 & 0.01951 & 0.00000 & 0.00000 & 0.00000 & 0.00390 & 0.00000 & 0.00390 & 0.00390 & 0.00000 & 0.00000 & 0.00000 & 0.00000 \\
\hline $\mathrm{H}_{2}$ gas & 0.00000 & 0.00000 & 0.00000 & 0.00000 & 0.00000 & 0.00000 & 0.00000 & 0.00000 & 0.00000 & 0.00000 & 0.00000 & 0.01000 & 0.00967 & 0.00033 & 0.00000 & 0.00000 & 0.00000 & 0.00000 & 0.00000 & 0.00000 & 0.00000 & 0.00000 \\
\hline 6 & 0.00000 & 0.00000 & 0.00000 & 0.00000 & 0.00000 & 0.00000 & 0.00000 & 0.00000 & 0.00000 & 0.00000 & 0.00000 & 0.00000 & 0.00000 & 0.00000 & 0.01577 & 0.00000 & 0.01577 & 0.00000 & 0.00000 & 0.00000 & 0.00000 & 0.00000 \\
\hline 7 & 0.00000 & 0.00000 & 0.00000 & 0.00000 & 0.00000 & 0.00000 & 0.00000 & 0.00000 & 0.00000 & 0.00000 & 0.00000 & 0.00000 & 0.00000 & 0.00000 & 0.00000 & 0.00000 & 0.00000 & 0.01880 & 0.00000 & 0.00000 & 0.00000 & 0.00000 \\
\hline IPA & 0.00000 & 0.00000 & 0.00000 & 0.00000 & 0.00000 & 0.00000 & 0.00000 & 0.00000 & 0.00000 & 0.00000 & 0.00000 & 0.00000 & 0.00000 & 0.00000 & 0.00000 & 0.00000 & 0.00000 & 0.00000 & 0.20000 & 0.01000 & 0.19000 & 0.00000 \\
\hline 8 & 0.00000 & 0.00000 & 0.00000 & 0.00000 & 0.00000 & 0.00000 & 0.00000 & 0.00000 & 0.00000 & 0.00000 & 0.00000 & 0.00000 & 0.00000 & 0.00000 & 0.00000 & 0.00000 & 0.00000 & 0.00000 & 0.00000 & 0.00000 & 0.00000 & 0.00000 \\
\hline 9 & 0.00000 & 0.00000 & 0.00000 & 0.00000 & 0.00000 & 0.00000 & 0.00000 & 0.00000 & 0.00000 & 0.00000 & 0.00000 & 0,00000 & 0.00000 & 0.00000 & 0.00000 & 0.00000 & 0.00000 & 0.00000 & 0.00000 & 0.00000 & 0.00000 & 0.00000 \\
\hline PivCl & 0.00000 & 0.00000 & 0.00000 & 0.00000 & 0.00000 & 0.00000 & 0.00000 & 0.00000 & 0.00000 & 0.00000 & 0.00000 & 0.00000 & 0.00000 & 0.00000 & 0.00000 & 0.00000 & 0.00000 & 0.00000 & 0.00000 & 0.00000 & 0.00000 & 0.00000 \\
\hline IPEA & 0.00000 & 0.00000 & 0.00000 & 0.00000 & 0.00000 & 0.00000 & 0.00000 & 0.00000 & 0.00000 & 0.00000 & 0.00000 & 0.00000 & 0.00000 & 0.00000 & 0.00000 & 0.00000 & 0.00000 & 0.00000 & 0.00000 & 0.00000 & 0.00000 & 0.00000 \\
\hline 10 & 0.00000 & 0.00000 & 0.00000 & 0.00000 & 0.00000 & 0.00000 & 0.00000 & 0.00000 & 0.00000 & 0.00000 & 0.00000 & 0.00000 & 0.00000 & 0.00000 & 0.00000 & 0.00000 & 0.00000 & 0.00000 & 0.00000 & 0.00000 & 0.00000 & 0.00000 \\
\hline by-product from R5 & 0.00000 & 0.00000 & 0.00000 & 0.00000 & 0.00000 & 0.00000 & 0.00000 & 0.00000 & 0.00000 & 0.00000 & 0.00000 & 0.00000 & 0.00000 & 0.00000 & 0.00000 & 0.00000 & 0.00000 & 0.00000 & 0.00000 & 0.00000 & 0.00000 & 0.00000 \\
\hline TFA & 0.00000 & 0.00000 & 0.00000 & 0.00000 & 0.00000 & 0.00000 & 0.00000 & 0.00000 & 0.00000 & 0.00000 & 0.00000 & 0.00000 & 0.00000 & 0.00000 & 0.00000 & 0.00000 & 0.00000 & 0.00000 & 0.00000 & 0.00000 & 0.00000 & 0.00000 \\
\hline 11 & 0.00000 & 0.00000 & 0.00000 & 0.00000 & 0.00000 & 0.00000 & 0.00000 & 0.00000 & 0.00000 & 0.00000 & 0.00000 & 0.00000 & 0.00000 & 0.00000 & 0.00000 & 0.00000 & 0.00000 & 0.00000 & 0.00000 & 0.00000 & 0.00000 & 0.00000 \\
\hline by-product from R6 & 0.00000 & 0.00000 & 0.00000 & 0.00000 & 0.00000 & 0.00000 & 0.00000 & 0.00000 & 0.00000 & 0.00000 & 0.00000 & 0.00000 & 0.00000 & 0.00000 & 0.00000 & 0.00000 & 0.00000 & 0.00000 & 0.00000 & 0.00000 & 0.00000 & 0.00000 \\
\hline DMSO & 0.00000 & 0.00000 & 0.00000 & 0.00000 & 0.00000 & 0.00000 & 0.00000 & 0.00000 & 0.00000 & 0.00000 & 0.00000 & 0.00000 & 0.00000 & 0.00000 & 0.00000 & 0.00000 & 0.00000 & 0.00000 & 0.00000 & 0.00000 & 0.00000 & 0.00000 \\
\hline$i-\mathrm{PrNH}_{2}$ & 0.00000 & 0.00000 & 0.00000 & 0.00000 & 0.00000 & 0.00000 & 0.00000 & 0.00000 & 0.00000 & 0.00000 & 0.00000 & 0.00000 & 0.00000 & 0.00000 & 0.00000 & 0.00000 & 0.00000 & 0.00000 & 0.00000 & 0.00000 & 0.00000 & 0.00000 \\
\hline 12 & 0.00000 & 0.00000 & 0.00000 & 0.00000 & 0.00000 & 0.00000 & 0.00000 & 0.00000 & 0.00000 & 0.00000 & 0.00000 & 0.00000 & 0.00000 & 0.00000 & 0.00000 & 0.00000 & 0.00000 & 0.00000 & 0.00000 & 0.00000 & 0.00000 & 0.00000 \\
\hline Acetone & 0.00000 & 0.00000 & 0.00000 & 0.00000 & 0.00000 & 0.00000 & 0.00000 & 0.00000 & 0.00000 & 0.00000 & 0.00000 & 0.00000 & 0.00000 & 0.00000 & 0.00000 & 0.00000 & 0.00000 & 0.00000 & 0.00000 & 0.00000 & 0.00000 & 0.00000 \\
\hline by-product from $\mathbf{R} 7$ & 0.00000 & 0.00000 & 0.00000 & 0.00000 & 0.00000 & 0.00000 & 0.00000 & 0.00000 & 0.00000 & 0.00000 & 0.00000 & 0.00000 & 0.00000 & 0.00000 & 0.00000 & 0.00000 & 0.00000 & 0.00000 & 0.00000 & 0.00000 & 0.00000 & 0.00000 \\
\hline $\mathrm{NaOH}$ & & & & & & & & & & & & & & & 0.00000 & & 0.00000 & & 0.00000 & 000 & 0.00000 & 0.00000 \\
\hline $\mathrm{NaCl}$ & 0.00000 & 0.00000 & 0.00000 & 0.00000 & 0.00000 & 0.00000 & 0.00000 & 0.00000 & 0.00000 & 0.00000 & 0.00000 & 0.00000 & 0.00000 & 0.00000 & 0.00000 & 0.00000 & 0.00000 & 0.00000 & 0.00000 & 0.00000 & 0.00000 & 0.00000 \\
\hline Brine & 0.00000 & 0.00000 & 0.00000 & 0.00000 & 0.00000 & 0.00000 & 0.00000 & 0.00000 & 0.00000 & 0.00000 & 0.00000 & 0.00000 & 0.00000 & 0.00000 & 0.00000 & 0.00000 & 0.00000 & 0.00000 & 0.00000 & 0.00000 & 0.00000 & 0.00000 \\
\hline Ethanol & 0.00000 & 0.00000 & 0.00000 & 0.00000 & 0.00000 & 0.00000 & 0.00000 & 0.00000 & 0.00000 & 0.00000 & 0.00000 & 0.00000 & 0.00000 & 0.00000 & 0.00000 & 0.00000 & 0.00000 & 0.00000 & 0.00000 & 0.00000 & .00000 & 0.00000 \\
\hline phoric & 0.00000 & 0.00000 & 0.00000 & 0.00000 & 0.00000 & 0.00000 & 0.00000 & 0.00000 & 0.00000 & 0.00000 & 0.00000 & 0.00000 & 0.00000 & 0.00000 & 0.00000 & 0.00000 & 0.00000 & 0.00000 & 0.00000 & 0.00000 & 0.00000 & 0.00000 \\
\hline 13 & 0.00000 & 0.00000 & 0.00000 & 0.00000 & 0.00000 & 0.00000 & 0.00000 & 0.00000 & 0.00000 & 0.00000 & 0.00000 & 0.00000 & 0.00000 & 0.00000 & 0.00000 & 0.00000 & 0.00000 & 0.00000 & 0.00000 & 0.00000 & 0.00000 & 0.00000 \\
\hline
\end{tabular}




\begin{tabular}{|c|c|c|c|c|c|c|c|c|c|c|c|c|c|c|c|c|c|c|c|c|c|c|}
\hline Mass now rate (kg/hr) & $\begin{array}{c}44 \\
\text { (solid) }\end{array}$ & $\begin{array}{c}44 \\
\text { (solution) }\end{array}$ & 45 & 46 & 47 & 48 & 49 & 50 & 51 & 52 & 53 & 54 & 55 & 56 & 57 & $\begin{array}{c}58 \\
\text { (solid) }\end{array}$ & $\begin{array}{c}58 \\
\text { (solution) }\end{array}$ & 59 & 60 & 61 & 62 & 63 \\
\hline 1 & 10000 & 0.00000 & 00000 & 0.00000 & 00000 & 0.00000 & 00000 & 00000 & 10000 & 00000 & 10000 & 00000 & 00000 & 00000 & .00000 & 0.00000 & 0.00000 & 0.00000 & 0.00000 & .00000 & 0.00000 & 0.00000 \\
\hline $\mathrm{H}_{2} \mathrm{C}$ & & 2857 & 2857 & & 2857 & 00000 & & 0000 & & 000 & 0000 & & & & 0.20000 & & 0.20000 & 0.30000 & 00000 & 0.10000 & 0.04086 & 0.04086 \\
\hline $\mathrm{NH}_{2} \mathrm{NH}_{2}$ & 0000 & 00000 & 00000 & 00000 & 00000 & 00000 & 00000 & 00000 & 00000 & 00000 & 00000 & 00000 & 00000 & 0.00000 & 0.00000 & 0.00000 & 0.00000 & 0.00000 & 0.00000 & 0.00000 & 0.00000 & 0.00000 \\
\hline 2 & 0000 & 0.00000 & 00000 & 00000 & 00000 & 00000 & 00000 & 00000 & 00000 & 00000 & .00000 & .00000 & 00000 & 0.00000 & 0.00000 & 0.00000 & 0.00000 & 0.00000 & 0.00000 & 0.00000 & .00000 & 0.00000 \\
\hline $\mathrm{HCl}$ & 00000 & 0.09698 & 09698 & 00000 & 09698 & 00000 & 00000 & 00000 & 00000 & 00000 & .00000 & 00000 & 00000 & 0.00214 & 0.00000 & 0.00000 & 0.00214 & 0.00214 & 0.00000 & 0.00000 & 0.00000 & 0.00000 \\
\hline 2-propanol & 00000 & 0.00000 & 00000 & 00000 & 00000 & 00000 & .00000 & 00000 & .00000 & 00000 & .00000 & .00000 & .00000 & 0.00000 & 0.00000 & 0.00000 & 0.00000 & 0.00000 & 0.00000 & 0.00000 & 0.00000 & 0.00000 \\
\hline dichlorometh & 0000 & 0.00000 & 0.00000 & 00000 & 00000 & 00000 & 0.00000 & 00000 & 0.00000 & 0.00000 & 0.00000 & 0.00000 & .00000 & 0.00000 & 0.00000 & 0.00000 & 0.00000 & 0.00000 & 0.00000 & 0.00000 & 0.00000 & 0.00000 \\
\hline IPAc & 0000 & 0.00000 & 00000 & 00000 & 00000 & 00000 & .00000 & 00000 & .00000 & 00000 & .00000 & .00000 & .00000 & 0.00000 & 0.00000 & & & & & & & 0.00000 \\
\hline 3 & 00000 & 0.00000 & 0.00000 & 0.00000 & 0.00000 & 0.00000 & 0.00000 & 0.00000 & 0.00000 & 0.00000 & 0.00000 & 0.00000 & 0.00000 & 0,00000 & 0.00000 & 0.00000 & 0.00000 & 0.00000 & 0.00000 & 0.00000 & 0.00000 & 0.00000 \\
\hline 4 & & 0.00348 & .00348 & 0.00000 & 00348 & 00000 & .00000 & 00000 & 00000 & 00000 & 00000 & .00000 & 00000 & 0.00000 & 00000 & . & 00000 & 0.00000 & . & 0.00000 & 0.00000 & 0.00000 \\
\hline Trifluoroacetic acid & 0.00000 & 1182 & 01182 & 0.00000 & 01182 & .00000 & 0.00000 & 0.00000 & 0.00000 & 0.00000 & 0.00000 & 0.00000 & 0.00000 & 0.00000 & 0.00000 & 0.00000 & 0.00000 & 0.00000 & 0.00000 & 0.00000 & 0.00000 & 0.00000 \\
\hline heptane & 0.00000 & 0.00000 & 0.00000 & 0.00000 & 0.00000 & 0.00000 & 0.00000 & 0.00000 & 0.00000 & 0.00000 & 0.00000 & 0.00000 & 0.00000 & 0.00000 & 0.00000 & 0.00000 & 0.00 & 0.000 & 0.00000 & 0.00000 & 0.00000 & 0.00000 \\
\hline Sup erphosph oric acid & 0.00000 & 0.05000 & 0.05000 & 0.00000 & 0.05000 & 0.00000 & 0.00000 & 0.00000 & 0.00000 & 0.00000 & 0.00000 & 0.00000 & 0.00000 & 0.00000 & 0.00000 & 0.00000 & 0.00000 & 0.00000 & 0.00000 & 0.00000 & 0.00000 & 0.00000 \\
\hline 5 & 00000 & 0.00390 & .00390 & 00000 & 0.00390 & .00000 & 0.00000 & .00000 & .00000 & 0.00000 & 0.00000 & 0.00000 & 0.00000 & 0.00000 & 0.00000 & 0.00000 & 0.00000 & 0.00000 & 0.00000 & 0.00000 & 0.00000 & 0.00000 \\
\hline $\mathrm{H}_{2} \mathrm{~g}$ & & & & & & 0000 & & & & 0000 & & 0000 & 00000 & 0000 & & & & & & & 000000 & \\
\hline 6 & 0.00000 & 0.00000 & .00000 & 0.00000 & 0.00000 & 0.00000 & 0.00000 & .00000 & 0.00000 & 0.00000 & 0.00000 & 0.00000 & 0.00000 & 0.00000 & 0.00000 & 0.00000 & 0.00000 & 0.00000 & 0.00000 & 0.00000 & 0.00000 & 0.00000 \\
\hline 7 & & & 0548 & 01331 & 0548 & 10000 & 0.00000 & 10000 & 0.00000 & 00000 & 0.00000 & 0.00000 & 0.01331 & 0.00000 & 0.00000 & 0.00000 & 0.00000 & 0.00000 & 0.00000 & 0.00000 & 0.00000 & 0.00000 \\
\hline IPA & & & & & & 00000 & 0.00000 & 00000 & 00000 & 0.00000 & 0.00000 & 0.00000 & 0.00000 & 0.00000 & 0.00000 & & 0.00000 & 0.00000 & & & & 0.00000 \\
\hline 8 & & & 0000 & 10000 & 0000 & 01176 & 00000 & 00000 & 0.00000 & 0.01176 & 0.00035 & .00000 & 0.00035 & 0.00035 & 0.00000 & 0.00000 & 0.00035 & 0.00035 & 0.00000 & 0.00000 & 0.00000 & 0.00000 \\
\hline 9 & & & & & 000 & & 00891 & .00000 & 0.00000 & & & .00 & & & & & & & & & & 0.00000 \\
\hline PivCl & & & 0000 & 0000 & 0000 & 0000 & & 0746 & 00000 & 0746 & & 00000 & & 0.00746 & .00000 & 0.00000 & & & 0.00000 & 0.00000 & 0.00000 & 0.00000 \\
\hline & & & & & & & & & & & & & & & & & & & & & & \\
\hline 10 & 0000 & 0.00000 & 00000 & 00000 & 00000 & 00000 & 00000 & 00000 & 10000 & 10000 & & 00000 & & .00000 & .00000 & 0.00000 & 0.00000 & 0.00000 & 0.00000 & 0.00000 & 0.00000 & 0.00000 \\
\hline by-product from R5 & 0.00000 & 00000 & 0.00000 & 0.00000 & 0.00000 & 0.00000 & 0.00000 & 0.00000 & 0.00000 & 0.00000 & 0.00129 & 0.00000 & 0.00129 & 0.00129 & 0.00000 & 0.00000 & 0.00129 & 0.00129 & 0.00000 & 0.00000 & 0.00000 & 0.00000 \\
\hline & & & & & & & & & & & & 01 & & & 1000 & & & & & & 9000 & 0.00000 \\
\hline 1 & & & & & & & & & & & & & & & & & & & & & & \\
\hline y-product fi & 10000 & 0000 & 10000 & 10000 & 0000 & 0000 & 0.00000 & 00000 & 00000 & 0.00000 & 00000 & 0.00000 & 00 & 0.00588 & .00000 & 0.00000 & 0.00588 & 0.00588 & 0.00000 & 0.00000 & 0.00000 & 0.00000 \\
\hline & & & 00000 & 00000 & 00000 & 00000 & 0.00000 & 00000 & 00000 & 00000 & 00000 & 0.00000 & 0.00000 & 0.00000 & .00000 & 0000 & .00000 & 0.10000 & 0.00000 & 0.10000 & 0.04086 & 0.04086 \\
\hline & & & & & & & & & & & & & & & & & & & & & & \\
\hline & & & & 0 & 000 & 000 & 00000 & 0000 & 00000 & 0000 & 00000 & 0000 & 00000 & 0000 & 00000 & & & 0000 & 00000 & 0.00000 & 000 & 0.00000 \\
\hline & & 00000 & 00000 & & 00000 & 00000 & 00000 & 00000 & 00000 & 10000 & & 00000 & 000 & 0000 & 0.00000 & 10000 & & 0.00000 & 1000 & 0.00000 & .00000 & 0.00000 \\
\hline by-product & & & & & & & & & & & & & & & & & & & & & & \\
\hline & & & & & & & & & & & & & & & & & & & & & & \\
\hline & & & & & & & & & & 10000 & & 00000 & & & & & & & & 000 & 000 & 0000 \\
\hline & & & & & & & & & & & & & & & & & & & & & & \\
\hline & & 0.00000 & & & & 00000 & 0.00000 & 00000 & 0.00000 & 00000 & & & & & & & & & & & 00000 & 0.00000 \\
\hline & & & & & & & & & & & & & & & & & & & & & & \\
\hline & 0.00000 & 0.00000 & 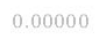 & 0.000000 & . & 0.00000 & 5.00000 & 0.00000 & 0.00000 & . & 80000 & . & 0.00000 & 0.0000 & D. & 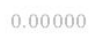 & . & 0.00000 & ond & 0.00000 & 0.00000 & . \\
\hline
\end{tabular}




\begin{tabular}{|c|c|c|c|c|c|c|c|c|c|c|c|c|c|c|c|c|c|c|c|c|c|c|}
\hline ass flow rate (kg/hr) & 64 & 65 & 66 & 67 & 68 & 69 & 70 & 71 & 72 & 73 & 74 & 75 & 76 & 77 & 78 & 79 & 80 & 81 & 82 & 83 & 84 & 35 \\
\hline 1 & & & 10000 & & & & & & & & & & & & & 0.00000 & & & & & & \\
\hline $\mathrm{H}_{2} \mathrm{O}$ & & 4086 & 02724 & 06810 & 06810 & 04086 & 00070 & 04156 & 00000 & 0000 & 00000 & 00000 & & & 0.04156 & 0.04156 & .00000 & 0.00000 & 00000 & 00000 & .00000 & 0.00000 \\
\hline $\mathrm{NH}_{2} \mathrm{NH}_{2}$ & & 0000 & 00000 & 00000 & 10000 & 00000 & 00000 & 0000 & 0.00000 & 00000 & 00000 & 0.00000 & 0.00000 & 0.00000 & 0.00000 & 0.00000 & 0.00000 & 0.00000 & 0.00000 & 0.00000 & 0.00000 & 0.00000 \\
\hline 2 & 0.00000 & 0000 & 00000 & 00000 & 0000 & 00000 & 00000 & 0000 & 00000 & 00000 & 00000 & 0.00000 & 00000 & 0.00000 & 0.00000 & 0.00000 & 0.00000 & 0.00000 & 0.00000 & 0.00000 & 0.00000 & 0.00000 \\
\hline $\mathrm{HCl}$ & 0000 & 0000 & 00000 & 0.00000 & 0.00000 & 0.00000 & 0.00030 & 0.00030 & .00000 & 0.00000 & 00000 & 0.00000 & 0.00000 & 0.00027 & 0.00003 & 0.00030 & 0.00027 & 0.00000 & 0.00000 & 0.00000 & 0.00000 & 0.00000 \\
\hline 2-propanol & 00000 & 0000 & 10000 & 00000 & 00000 & 00000 & 00000 & 00000 & 00000 & 00000 & 00000 & 0.00000 & .00000 & 0.00000 & 0.00000 & 0.00000 & 0.00000 & 0.00000 & 0.00000 & 0.00000 & 0.00000 & 0.00000 \\
\hline lorometh & 10000 & 00000 & 00000 & 0.00000 & 00000 & 0.00000 & 00000 & 0000 & 00000 & 0.00000 & .00000 & 0.00000 & 0.00000 & 0.00000 & 0.00000 & 0.00000 & 0.00000 & 0.00000 & 0.00000 & 0.00000 & 0.00000 & 0.00000 \\
\hline IPAc & 10000 & 0000 & 0000 & 00000 & 0000 & 00000 & 00000 & 0000 & 20000 & 01190 & 0.18810 & 00190 & 0.19000 & 0.20000 & 0.00000 & 0.20000 & 0.01000 & 0.00400 & 0.36000 & .36400 & 0.20000 & 0.16400 \\
\hline & & 0000 & 0000 & 60000 & 10000 & 00000 & 10000 & 0000 & & 00000 & & 00000 & 100000 & & 0.00000 & 0.00000 & 0.00000 & 0.00000 & 0.00000 & 00000 & 0.00000 & 00000 \\
\hline 4 & 0.00000 & .00000 & 00000 & .00000 & 00000 & 00000 & 00000 & 0000 & .00000 & 00000 & .00000 & 0.00000 & 0.00000 & 0.00000 & 0.00000 & 0.00000 & 0.00000 & 0.00000 & 0.00000 & .00000 & 0.00000 & 0.00000 \\
\hline Trifluoroacet: & 0.00000 & 00000 & 00000 & 0.00000 & 00000 & 00000 & 00000 & 0000 & 00000 & 10000 & 00000 & 50000 & 0.00000 & . & 0.00000 & 0.00000 & 0.00000 & 0.00000 & 0.00000 & .00000 & 0.00000 & 0.00000 \\
\hline e & 00000 & 0000 & 0000 & 00000 & 10000 & 10000 & 0000 & 00 & 000 & 00 & & & & 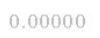 & 0.000 & 0.000 & 0.000 & 0.00000 & 0.000 & 0.00 & 0.000 & 0.000 \\
\hline Sup erph osphoric acid & 00000 & 00000 & 0.00000 & 0.00000 & 00000 & 0.00000 & 00000 & 0.00000 & 0.00000 & 0.00000 & 0.00000 & 0.000 & 0.00000 & 0.000 & 0.00000 & 0.00000 & 0.00000 & 0.000 & 0.00000 & 0.00 & 0.00000 & 0.00 \\
\hline & & 2000 & 000 & 0.000 & 600 & .0000 & 10 & 000 & 0.00 & 00 & & & & 0.00 & 0.00000 & 0.000 & 0.00000 & 0.00 & 0.00000 & 0.00 & 0.00000 & 0.00000 \\
\hline $\mathrm{H}_{2}$ gas & 0000 & 00000 & 00000 & .00000 & 00000 & 00000 & 00000 & 00000 & .00000 & 00000 & .00000 & 0.00000 & 0.00000 & 0.00000 & 0.00000 & 0.00000 & 0.00000 & 0.00000 & 0.00000 & .00000 & 0.00000 & 0.00000 \\
\hline & & & & 0 & & 0000 & & & & & & & & & & & & & & & & \\
\hline 7 & 0000 & 00000 & 00000 & 00000 & 00000 & 00000 & 00000 & 00000 & .00000 & 00000 & 00000 & 0.00000 & 0.00000 & 0.00000 & 0.00000 & 0.00000 & 0.00000 & 0.00000 & 0.00000 & .00000 & 0.00000 & 0.00000 \\
\hline IP & & & & & & & & & & & & & & & & & & & & & & 00000 \\
\hline & 000 & 0000 & 10000 & 00000 & 0000 & 00000 & 0000 & 00000 & 000 & 00000 & 0.00000 & 0.00000 & 0.00000 & 0.00000 & 0.00000 & 0.00000 & 0.00000 & 0.00000 & 0.00000 & .00000 & 0.00000 & 0.00000 \\
\hline & & 0000 & 1000 & 0.00000 & 0000 & 10000 & 0000 & 10000 & & 000 & & & & 00000 & 0.00000 & 0.000000 & 0.00000 & 0.00000 & 0.00000 & 100000 & 0.00000 & 0.00000 \\
\hline & & & & & & 0 & & & & & & & & & & & & & & & & \\
\hline & 0000 & 10000 & 0000 & 0.00000 & 0000 & 00000 & 0000 & 10000 & 0000 & 00000 & 0000 & 10000 & 0.00000 & 00000 & 0.00000 & .00000 & 0.00000 & 0.00000 & 0.00000 & .00000 & 0.00000 & 0.00000 \\
\hline & & & & 00000 & & 10000 & & & & & & & & & & & & & & & & 0.00000 \\
\hline by-product fr & 0.00000 & 00000 & 00000 & 0.00000 & 00000 & 0000 & 00000 & 00000 & 0.00000 & 00000 & 0.00000 & 00000 & 0.00000 & 0.00000 & 0.00000 & 0.00000 & 0.00000 & 0.00000 & 0.00000 & .00000 & 0.00000 & 0.00000 \\
\hline TFA & onos & 0.00000 & 10000 & 0.00000 & 00000 & 00000 & 0000 & 00000 & 1000 & 10000 & & & & 0.00000 & 0.00000 & 0.00000 & 0.00000 & 0.00000 & 0.00000 & 0.00000 & 0.00000 & 0.00000 \\
\hline 11 & & 0.02111 & 0001 & 22112 & 0003 & 0002 & & 00002 & & 00000 & & & & 0.00002 & 0.00000 & 0.00002 & 0.00002 & 00000 & 0.00000 & .00000 & 0.00000 & 0.00000 \\
\hline & 0.0000 & 00000 & 0000 & 0000 & & 0000 & & & & & & & & & 0.0 & & 0.00000 & & & & 0.00000 & 0.00000 \\
\hline & & & & & & & & & & & & & & & & & & & & & & .00000 \\
\hline & 03074 & 74 & & & & & & & & 100 & & & & & & & & & 0.00000 & 00000 & 0.00000 & .00000 \\
\hline & & & & & & & & & & & & & & & & & & & & & & \\
\hline Acetone & 0.00000 & 0.00000 & 0105 & & & & 10000 & & 0.00000 & 00000 & 000 & 0000 & 1000 & 00141 & 0.00016 & & 0.00141 & 00000 & 0.00000 & .00000 & 0.00000 & 0.00000 \\
\hline by-product from $\mathbf{R} 7$ & 0.00000 & 0.00000 & 0.00405 & 0.00405 & 01011 & 0.00607 & 0.00000 & 0.00607 & 0.00000 & 0.00000 & 0.00000 & 0.00000 & 0.00000 & 0.00607 & 0.00000 & 0.00607 & 0.00607 & 0.00000 & 0.00000 & 0.00000 & 0.00000 & 0.00000 \\
\hline & & & & & & & & & & & & & & & & & & & & & & \\
\hline & & & & & & & & & & & & & & & & & & & & & & \\
\hline & 0. & 00000 & 00000 & 0.000 & & 10000 & 000 & & & & & & & & & & & & & 000 & 0.00000 & .00000 \\
\hline & & & & & & & & & & & & & & & & & & & & & & \\
\hline Phosph oric & 0.00000 & 0.00000 & 00000 & 0.00000 & 00000 & 0.00000 & 00000 & & 0.00000 & & 0.00000 & & & & & & & & & 00000 & .00000 & 00000 \\
\hline 13 & & & & & & & & & & & & & & & & & & & & & & \\
\hline
\end{tabular}




\begin{tabular}{|c|c|c|c|c|c|c|c|c|c|c|c|c|c|c|c|c|c|c|c|c|c|c|}
\hline Mass now rate $(\mathrm{kg} / \mathrm{hr})$ & 86 & 87 & 88 & 89 & 90 & 91 & 92 & 93 & 94 & 95 & 96 & 97 & 98 & 99 & 100 & 101 & 102 & 103 & $\begin{array}{c}104 \\
\text { (anhydrous } \\
\mathrm{Na}_{2} \mathrm{SO}_{4} \text { ) }\end{array}$ & 105 & 106 & 107 \\
\hline 1 & 0.00000 & 00000 & 0.00000 & 0.00000 & 0.00000 & 0.00000 & 0.00000 & 0.00000 & 0.00000 & 0.00000 & 0.00000 & 0.00000 & 0.00000 & 0.00000 & 0.00000 & 0.00000 & .00000 & 0.00000 & 0.00000 & 0.00000 & 0,00000 & 0.00000 \\
\hline $\mathrm{H}_{2} \mathrm{O}$ & 0.00000 & 0.00000 & 0.00144 & 0.04300 & 0.04300 & 0.00000 & 0.04300 & 0.04300 & 0.04300 & 0.00000 & 0.00000 & 0.00000 & 0.00000 & 0.00000 & 0.00000 & 0.00000 & 0.00000 & 0.00000 & 0.00000 & 0.00000 & 0.00000 & 0.00000 \\
\hline $\mathrm{NH}_{2} \mathrm{NH}_{2}$ & 0.00000 & 0.00000 & 0.00000 & 0.00000 & 0.00000 & 0.00000 & 0.00000 & 0.00000 & 0.00000 & 0.00000 & 0.00000 & 0.00000 & 0.00000 & 0.00000 & 0.00000 & 0.00000 & 0.00000 & 0.00000 & 0.00000 & 0.00000 & 0.00000 & 0.00000 \\
\hline 2 & 0.00000 & 0.00000 & 0.00000 & 0.00000 & 0.00000 & 0.00000 & 0.00000 & 0.00000 & 0.00000 & 0.00000 & 0,00000 & 0.00000 & 0.00000 & 0.00000 & 0.00000 & 0.00000 & 0.00000 & 0.00000 & 0.00000 & 0.00000 & 0.00000 & 0.00000 \\
\hline $\mathrm{HCl}$ & 0.00000 & 0.00000 & 0.00000 & 0.00000 & 0.00000 & 0.00000 & 0.00000 & 0.00000 & 0.00000 & 0.00000 & 0.00000 & 0.00000 & 0.00000 & 0.00000 & 0.00000 & 0.00000 & 0.00000 & 0.00000 & 0.00000 & 0.00000 & 0.00000 & 0.00000 \\
\hline 2-propanol & 0.00000 & 0.00000 & 0.00000 & 0.00000 & 0.00000 & 0.00000 & 0.00000 & 0.00000 & 0.00000 & 0.00000 & 0.00000 & 0.00000 & 0.00000 & 0.00000 & 0.00000 & 0.00000 & 0.00000 & 0.00000 & 0.00000 & 0.00000 & 0.00000 & 0.00000 \\
\hline dichloromethane & 0.00000 & 0.00000 & 0.00000 & 0.00000 & 0.00000 & 0.00000 & 0.00000 & 0.00000 & 0.00000 & 0.00000 & 0.00000 & 0.00000 & 0.00000 & 0.00000 & 0.00000 & 0.00000 & 0.00000 & 0.00000 & 0.00000 & 0.00000 & 0.00000 & 0.00000 \\
\hline IPAc & 0.20000 & 0.03600 & 0.00000 & 0.00000 & 0.20000 & 0.20000 & 0.00000 & 0.20000 & 0.00000 & 0.20000 & 0.40000 & 0.00000 & 0.00000 & 0.00000 & 0.00000 & 0.00000 & 0.40000 & 0.40000 & 0.00000 & 0.40000 & 0.04000 & 0.00000 \\
\hline 3 & 0.00000 & 0.00000 & 0.00000 & 0.00000 & 0.00000 & 0.00000 & 0.00000 & 0.00000 & 0.00000 & 0.00000 & 0.00000 & 0.00000 & 0.00000 & 0.00000 & 0.00000 & 0.00000 & 0.00000 & 0.00000 & 0.00000 & 0.00000 & 0.00000 & 0.00000 \\
\hline 4 & 0.00000 & 0.00000 & 0.00000 & 0.00000 & 0.00000 & 0.00000 & 0.00000 & 0.00000 & 0.00000 & 0.00000 & 0.00000 & 0.00000 & 0.00000 & 0.00000 & 0.00000 & 0.00000 & 0.00000 & 0.00000 & 0.00000 & 0.00000 & 0.00000 & 0.00000 \\
\hline Trifluoroacetic acid & 0.00000 & 0.00000 & 0.00000 & 0.00000 & 0.00000 & 0.00000 & 0.00000 & 0.00000 & 0.00000 & 0.00000 & 0.00000 & 0.00000 & 0.00000 & 0.00000 & 0.00000 & 0.00000 & 0.00000 & 0.00000 & 0.00000 & 0.00000 & 0.00000 & 0.00000 \\
\hline heptane & 0.00000 & 0.00000 & 0.00000 & 0.00000 & 0.00000 & 0.00000 & 0.00000 & 0.00000 & 0.00000 & 0.00000 & 0.00000 & 0.00000 & 0.00000 & 0.00000 & 0.00000 & 0.00000 & 0.00000 & 0.00000 & 0.00000 & 0.00000 & 0.00000 & 0.00000 \\
\hline Sup erphosphoric acid & 0.00000 & 0.00000 & 0.00000 & 0.00000 & 0.00000 & 0.00000 & 0.00000 & 0.00000 & 0.00000 & 0.00000 & 0.00000 & 0.00000 & 0.00000 & 0.00000 & 0.00000 & 0.00000 & 0.00000 & 0.00000 & 0.00000 & 0.00000 & 0.00000 & 0.00000 \\
\hline 5 & 0.00000 & 0.00000 & 0.00000 & 0.00000 & 0.00000 & 0.00000 & 0.00000 & 0.00000 & 0.00000 & 0.00000 & 0.00000 & 0.00000 & 0.00000 & 0.00000 & 0.00000 & 0.00000 & 0.00000 & 0.00000 & 0.00000 & 0.00000 & 0.00000 & 0.00000 \\
\hline $\mathrm{H}_{2}$ gas & 0.00000 & 0.00000 & 0.00000 & 0.00000 & 0.00000 & 0.00000 & 0.00000 & 0.00000 & 0.00000 & 0.00000 & 0.00000 & 0.00000 & 0.00000 & 0.00000 & 0.00000 & 0.00000 & 0.00000 & 0.00000 & 0.00000 & 0.00000 & 0.00000 & 0.00000 \\
\hline 6 & 0.00000 & 0.00000 & 0.00000 & 0.00000 & 0.00000 & 0.00000 & 0.00000 & 0.00000 & 0.00000 & 0.00000 & 0.00000 & 0.00000 & 0.00000 & 0.00000 & 0.00000 & 0.00000 & 0.00000 & 0.00000 & 0.00000 & 0.00000 & 0.00000 & 0.00000 \\
\hline 7 & 0.00000 & 0.00000 & 0.00000 & 0.00000 & 0.00000 & 0.00000 & 0.00000 & 0.00000 & 0.00000 & 0.00000 & 0.00000 & 0.00000 & 0.00000 & 0.00000 & 0.00000 & 0.00000 & 0.00000 & 0.00000 & 0.00000 & 0.00000 & 0.00000 & 0.00000 \\
\hline IPA & 0.00000 & 0.00000 & 0.00000 & 0.00000 & 0.00000 & 0.00000 & 0.00000 & 0.00000 & 0.00000 & 0.00000 & 0.00000 & 0.00000 & 0.00000 & 0.00000 & 0.00000 & 0.00000 & 0.00000 & 0.00000 & & 0.00000 & 0.00000 & 0.00000 \\
\hline 8 & 0.00000 & 0.00000 & 0.00000 & 0.00000 & 0.00000 & 0.00000 & 0.00000 & 0.00000 & 0.00000 & 0.00000 & 0.00000 & 0.00000 & 0.00000 & 0.00000 & 0.00000 & 0.00000 & 0.00000 & 0.00000 & 0.00000 & 0.00000 & 0.00000 & 0.00000 \\
\hline 9 & 0.00000 & 0.00000 & 0.00000 & 0.00000 & 0.00000 & 0.00000 & 0.00000 & 0.00000 & 0.00000 & 0.00000 & 0.00000 & 0.00000 & 0.00000 & 0.00000 & 0.00000 & 0.00000 & 0.00000 & 0.00000 & & 0.00000 & 0.00000 & 0.00000 \\
\hline PivCl & 0.00000 & 0.00000 & 0.00000 & 0.00000 & 0.00000 & 0.00000 & 0.00000 & 0.00000 & 0.00000 & 0.00000 & 0.00000 & 0.00000 & 0.00000 & 0.00000 & 0.00000 & 0.00000 & 0.00000 & 0.00000 & 0.00000 & 0.00000 & 0.00000 & 0.00000 \\
\hline IPEA & & 0.00000 & 0.00000 & 0.00000 & 0.00000 & 0.00000 & 0.00000 & 0.00000 & 0.00000 & 0.00000 & 0.00000 & 0.00000 & 0.00000 & 0.00000 & 0.00000 & 0.00000 & 0.00000 & 0.00000 & & 0.00000 & 0.00000 & 0.00000 \\
\hline 10 & 0.00000 & 0.00000 & 0.00000 & 0.00000 & 0.00000 & 0.00000 & 0.00000 & 0.00000 & 0.00000 & 0.00000 & 0.00000 & 0.00000 & 0.00000 & 0.00000 & 0.00000 & 0.00000 & 0.00000 & 0.00000 & 0.00000 & 0.00000 & 0.00000 & 0.00000 \\
\hline by-product from R5 & 0.00000 & 0.00000 & 0.00000 & 0.00000 & 0.00000 & 0.00000 & 0.00000 & 0.00000 & 0.00000 & 0.00000 & 0.00000 & 0.00000 & 0.00000 & 0.00000 & 0.00000 & 0.00000 & 0.00000 & 0.00000 & 0.00000 & 0.00000 & 0.00000 & 0.00000 \\
\hline TFA & 0.00000 & 0.00000 & 0.00000 & 0.00000 & 0.00000 & 0.00000 & 0.00000 & 0.00000 & 0.00000 & 0.00000 & 0.00000 & 0.00000 & 0.00000 & 0.00000 & 0.00000 & 0.00000 & 0.00000 & 0.00000 & 0.00000 & 0.00000 & 0.00000 & 0.00000 \\
\hline 11 & & 0.00000 & 0.00000 & 0.00000 & & 10000 & 0.00000 & .00000 & 0000 & 0000 & 0.00000 & 0.00000 & 0.00000 & 0000 & 0.00000 & 0.00000 & 0.00000 & 0.00000 & & 0.00000 & 0.00000 & 0.00000 \\
\hline by-product from R6 & 0.00000 & 0.00000 & 0.00000 & 0.00000 & 0.00000 & 0.00000 & 0.00000 & 0.00000 & 0.00000 & 0.00000 & 0.00000 & 0.00000 & 0.00000 & 0.00000 & 0.00000 & 0.00000 & 0.00000 & 0.00000 & 0.00000 & 0.00000 & 0.00000 & 0.00000 \\
\hline DMSO & 0.00000 & 0.00000 & 0.00000 & 0.00409 & 0.00409 & 0.00368 & 0.00041 & 0.00041 & 0.00004 & 0.00037 & 0.40451 & 0.40451 & 0.00405 & 0.40047 & 0.00000 & 0.00000 & 0.00000 & 0.00000 & 0.00000 & 0.00000 & 0.00000 & 0.00000 \\
\hline$i-\mathrm{PrNH}_{2}$ & & 0.00000 & 0.00000 & 0.01898 & & 0.01708 & 00190 & 00190 & & & & 1.87895 & 0.01879 & & 0.00000 & 0.00000 & 0.00000 & 0.00000 & 000 & 0.00000 & 0.00000 & 0.00000 \\
\hline 12 & 0.00000 & 0.00000 & 0.00000 & 0.01002 & 002 & 0.00781 & 0220 & 00220 & 0.00013 & 0207 & 1097 & 00110 & .00001 & 109 & 0.00000 & 0.00000 & 0.00987 & 0.00987 & 0.00000 & 0.00987 & 0.00987 & 0.00000 \\
\hline Acetone & 0.00000 & 0.00000 & 0.00000 & 0.00016 & 0.00016 & 0.00014 & 0.00002 & 0.00002 & 0.00000 & 0.00001 & 0.01554 & 0.01554 & 0.00016 & 0.01538 & 0.00000 & 0.00000 & 0.00000 & 0.00000 & 0.00000 & 0.00000 & 0.00000 & 0.00000 \\
\hline by-product from $\mathbf{R} 7$ & 0.00000 & 0.00000 & 0.00000 & 0.00000 & 0.00000 & 0.00000 & 0.00000 & 0.00000 & 0.00000 & 0.00000 & 00000 & 0.00000 & 0.00000 & 0.00000 & 0.00000 & 0.00000 & 0.00000 & 0.00000 & 0000 & 0.00000 & 0.00000 & 0.00000 \\
\hline $\mathrm{NaOH}$ & & & 0.00056 & & & & & & & & & & & & & & & & & & 0.00000 & 0.00000 \\
\hline $\mathrm{NaCl}$ & 0.00000 & 0.00000 & 0.00000 & 0.00005 & 0.00005 & 0.00004 & 0.00000 & 0.00000 & 0.00000 & 0.00000 & 0.00483 & 0.00483 & 0.00005 & 00478 & 0.00000 & 0.00000 & 0.00000 & 0.00000 & 0.00000 & 0.00000 & 0.00000 & 0.00000 \\
\hline Brine & & 0.00000 & 0.00000 & 0.00000 & 0.00000 & 0.00000 & 0.00000 & 0.00000 & 0.00000 & & 0.10000 & 0.10000 & 0.00100 & 09900 & 0.00100 & 0.10000 & 0.00000 & 0.00000 & 0.00000 & 0.00000 & 0.00000 & 0.00000 \\
\hline Ethanol & 0.00000 & 0.00000 & 0.00000 & 0.00000 & 0.00000 & 0.00000 & 0.00000 & 0.00000 & 0.00000 & 0.00000 & 0.00000 & 0.00000 & 0.00000 & 0.00000 & 0.00000 & 0.00000 & 0.00000 & 0.00000 & 0.00000 & 0.00000 & 0.00000 & 0.20000 \\
\hline sphoric a & & & & & & & & & & & & 0.00000 & 1000 & 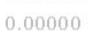 & & 1000 & 0.00000 & 0000 & 0.00000 & 0.00000 & 0.00000 & 0.00000 \\
\hline 13 & 0.00000 & 0.00000 & 0.00000 & 0.00000 & 0.00000 & 0.00000 & 0.00000 & 0.00000 & 0.00000 & 0.00000 & 0.00000 & 0.00000 & 0.00000 & 0.00000 & 0.00000 & 0.00000 & 0.00000 & 0.00000 & 0.00000 & 0.00000 & 0.00000 & 0.00000 \\
\hline
\end{tabular}




\begin{tabular}{|c|c|c|c|c|c|c|c|c|c|c|c|c|c|c|c|c|c|}
\hline Mass flow rate (kg $/ \mathrm{hr})$ & 108 & 109 & 110 & 111 & 112 & 113 & $\begin{array}{c}114 \\
\text { (solid) }\end{array}$ & $\begin{array}{c}114 \\
\text { (solution) }\end{array}$ & 115 & 116 & 117 & 118 & 119 & 120 & 121 & 122 & 123 \\
\hline 1 & 0.00000 & 0.00000 & 0.00000 & 0.00000 & 0,00000 & 0.00000 & 0.00000 & 0.00000 & 0.00000 & 0.00000 & 0.00000 & 0.00000 & 0.00000 & 0.00000 & 0.00000 & 0.00000 & 0.00000 \\
\hline $\mathrm{H}_{2} \mathrm{O}$ & 0.00000 & 0.00000 & 0.00000 & 0.00126 & 0.00126 & 0.00126 & 0.00000 & 0.00126 & 0.00126 & 0.00126 & 0.00000 & 0.00000 & 0.00000 & 0.00000 & 0.00000 & 0.00000 & 0.00000 \\
\hline $\mathrm{NH}_{2} \mathrm{NH}_{2}$ & 0.00000 & 0.00000 & 0.00000 & 0.00000 & 0.00000 & 0.00000 & 0.00000 & 0.00000 & 0.00000 & 0.00000 & 0.00000 & 0.00000 & 0.00000 & 0.00000 & 0.00000 & 0.00000 & 0.00000 \\
\hline 2 & 0.00000 & 0.00000 & 0.00000 & 0.00000 & 0.00000 & 0.00000 & 0.00000 & 0,00000 & 0.00000 & 0.00000 & 0.00000 & 0.00000 & 0.00000 & 0.00000 & 0.00000 & 0.00000 & 0.00000 \\
\hline $\mathrm{HCl}$ & 0.00000 & 0.00000 & 0.00000 & 0.00000 & 0.00000 & 0.00000 & 0.00000 & 0.00000 & 0.00000 & 0.00000 & 0.00000 & 0.00000 & 0.00000 & 0.00000 & 0.00000 & 0.00000 & 0.00000 \\
\hline 2-propanol & 0.00000 & 0.00000 & 0.00000 & 0.00000 & 0.00000 & 0.00000 & 0.00000 & 0,00000 & 0.00000 & 0.00000 & 0.00000 & 0.00000 & 0.00000 & 0.00000 & 0.00000 & 0.00000 & 0.00000 \\
\hline dichloromethane & 0.00000 & 0.00000 & 0.00000 & 0.00000 & 0.00000 & 0.00000 & 0.00000 & 0.00000 & 0.00000 & 0.00000 & 0.00000 & 0.00000 & 0.00000 & 0.00000 & 0.00000 & 0.00000 & 0.00000 \\
\hline IPAc & 0.00000 & 0.00000 & 0.04000 & 0.00000 & 0.04000 & 0.04000 & 0.00000 & 0.04000 & 0.04000 & 0.00400 & 0.00000 & 0.00000 & 0.00000 & 0.00000 & 0.00000 & 0.00000 & 0.00000 \\
\hline 3 & 0.00000 & 0.00000 & 0.00000 & 0.00000 & 0.00000 & 0.00000 & 0.00000 & 0.00000 & 0.00000 & 0.00000 & 0.00000 & 0.00000 & 0.00000 & 0.00000 & 0.00000 & 0.00000 & 0.00000 \\
\hline 4 & 0.00000 & 0.00000 & 0.00000 & 0.00000 & 0.00000 & 0.00000 & 0.00000 & 0.00000 & 0.00000 & 0.00000 & 0.00000 & 0.00000 & 0.00000 & 0.00000 & 0.00000 & 0.00000 & 0.00000 \\
\hline Trifluoroacetic acid & 0.00000 & 0.00000 & 0.00000 & 0.00000 & 0.00000 & 0.00000 & 0.00000 & 0.00000 & 0.00000 & 0.00000 & 0.00000 & 0.00000 & 0.00000 & 0.00000 & 0.00000 & 0.00000 & 0.00000 \\
\hline heptane & 0.00000 & 0.00000 & 0.00000 & 0.00000 & 0.00000 & 0.00000 & 0.00000 & 0.00000 & 0.00000 & 0.00000 & 0.00000 & 0.00000 & 0.00000 & 0.00000 & 0.00000 & 0.00000 & 0.00000 \\
\hline Sup erphosph oric acid & 0.00000 & 0.00000 & 0.00000 & 0.00000 & 0.00000 & 0.00000 & 0.00000 & 0.00000 & 0.00000 & 0.00000 & 0.00000 & 0.00000 & 0.00000 & 0.00000 & 0.00000 & 0.00000 & 0.00000 \\
\hline 5 & 0.00000 & 0.00000 & 0.00000 & 0.00000 & 0.00000 & 0.00000 & 0.00000 & 0.00000 & 0,00000 & 0.00000 & 0.00000 & 0.00000 & 0.00000 & 0.00000 & 0.00000 & 0.00000 & 0.00000 \\
\hline $\mathrm{H}_{2}$ gas & 0.00000 & 0.00000 & 0.00000 & 0.00000 & 0.00000 & 0.00000 & 0.00000 & 0.00000 & 0.00000 & 0.00000 & 0.00000 & 0.00000 & 0.00000 & 0.00000 & 0.00000 & 0.00000 & 0.00000 \\
\hline 6 & 0.00000 & 0.00000 & 0.00000 & 0.00000 & 0.00000 & 0.00000 & 0.00000 & 0.00000 & 0.00000 & 0.00000 & 0.00000 & 0.00000 & & 0.00000 & 00000 & 0.00000 & 0.00000 \\
\hline 7 & 0.00000 & 0.00000 & 0.00000 & 0.000 & 0.00000 & 0.000 & & & 0.000 & 0.000 & 0.000 & 0.00000 & 00 & 0.00000 & 00 & 0.00000 & 0.00000 \\
\hline IPA & 0.00000 & 0.00000 & 0.00000 & 0.00000 & 0.00000 & 0.00000 & 0.00000 & 0.00000 & 0.00000 & 0.00000 & 0,00000 & 0.00000 & 0.00000 & 0.00000 & 0.00000 & 0.00000 & 0.00000 \\
\hline 8 & 0.00000 & 0.00000 & 0.00000 & 0.00000 & 0.00000 & 0.00000 & 0.00000 & 0.00000 & 0.00000 & 0.00000 & 0.00000 & 0.00000 & 0.00000 & 0.00000 & 0.00000 & 0.00000 & 0.00000 \\
\hline 9 & 0.00000 & 0.00000 & 0.00000 & 0.00000 & 0.00000 & 0.00000 & 0.00000 & 0.00000 & 0.00000 & 0.00000 & 0.00000 & 0.00000 & 0.00000 & 0.00000 & 0.00000 & 0.00000 & 0.00000 \\
\hline PivCl & 0.00000 & 0.00000 & 0.00000 & 0.00000 & 0.00000 & 0.00000 & 0.00000 & 0.00000 & 0.00000 & 0.00000 & 0.00000 & 0.00000 & 0.00000 & 0.00000 & 0.00000 & 0.00000 & 0.00000 \\
\hline IPEA & 0.00000 & 0.00000 & 0.00000 & 0.00000 & 0.00000 & 0.00000 & 0.00000 & 0.00000 & 0.00000 & 0.00000 & 0.00000 & 0.00000 & 0.00000 & 0.00000 & 0.00000 & 0.00000 & 0.00000 \\
\hline 10 & 0.00000 & 0.00000 & 0.00000 & 0.00000 & 0.00000 & 0.00000 & 0.00000 & 0.00000 & 0.00000 & 0.00000 & 0.00000 & 0.00000 & 0.00000 & 0.00000 & 0.00000 & 0.00000 & 0.00000 \\
\hline by-product from R5 & 0.00000 & 0.00000 & 0.00000 & 0.00000 & 0.00000 & 0.00000 & 0.00000 & 0.00000 & 0.00000 & 0.00000 & 0.00000 & 0.00000 & 0.00000 & 0.00000 & 0.00000 & 0.00000 & 0.00000 \\
\hline TFA & 0.00000 & 0.00000 & 0.00000 & 0.00000 & 0.00000 & 0.00000 & 0.00000 & 0.00000 & 0.00000 & 0.00000 & 0.00000 & 0.00000 & 0,00000 & 0.00000 & 0.00000 & 0.00000 & 0.00000 \\
\hline 11 & 0.00000 & 0.00000 & 0.00000 & 0.00000 & 0.00000 & 0.00000 & 0.00000 & 0.00000 & 0.00000 & 0.00000 & 0.00000 & 0.00000 & 0.00000 & 0.00000 & 0.00000 & 0.00000 & 0.00000 \\
\hline by-product from R6 & 0.00000 & 0.00000 & 0.00000 & 0.00000 & 0.00000 & 0.00000 & 0.00000 & 0.00000 & 0.00000 & 0.00000 & 0.00000 & 0.00000 & 0.00000 & 0.00000 & 0.00000 & 0.00000 & 0.00000 \\
\hline DMSO & 0.00000 & 0.00000 & 0.00000 & 0.00000 & 0.00000 & 0.00000 & 0.00000 & 0.00000 & 0.00000 & 0.00000 & 0.00000 & 0.00000 & 0.00000 & 0.00000 & 0.00000 & 0.00000 & 0.00000 \\
\hline$i-\mathrm{PrNH}_{2}$ & 0.00000 & 0.00000 & 0.00000 & 0.00000 & 0.00000 & 0.00000 & 0.00000 & 0.00000 & 0.00000 & 0.00000 & 0.00000 & 0.00000 & 0.00000 & 0.00000 & 0.00000 & 0.00000 & 0.00000 \\
\hline 12 & 0.00000 & 0.00000 & 0.00987 & 0.00000 & 0.00987 & 0.00000 & 0.00000 & 0.00000 & 0.00000 & 0.00000 & 0.00000 & 0.00000 & 0.00000 & 0.00000 & 0.00000 & 0.00000 & 0.00000 \\
\hline Acetone & 0.00000 & 0.00000 & 0.00000 & 0.00000 & 0.00000 & 0.00000 & 0.00000 & 0.00000 & 0.00000 & 0.00000 & 0.00000 & 0.00000 & 0.00000 & 0.00000 & 0.00000 & 0.00000 & 0.00000 \\
\hline by-product from $\mathbf{R} 7$ & 0.00000 & 0.00000 & 0.00000 & 0.00000 & 0.00000 & 0.00000 & 0.00000 & 0.00000 & 0.00000 & 0.00000 & 0.00000 & 0.00000 & 0.00000 & 0.00000 & 0.00000 & 0.00000 & 0.00000 \\
\hline $\mathrm{NaOH}$ & 0.00000 & 0.00000 & 0.00000 & 0.00000 & 0.00000 & 0.00000 & 0.00000 & 0.00000 & 0.00000 & 0.00000 & 0.00000 & 0.00000 & 0.00000 & 0.00000 & 0.00000 & 0.00000 & 0.00000 \\
\hline $\mathrm{NaCl}$ & 0.00000 & 0.00000 & 0.00000 & 0.00000 & 0.00000 & 0.00000 & 0000 & 0.00000 & 0.00000 & 0.00000 & 0.00000 & 0.00000 & 0.00000 & 0.00000 & 0.00000 & 0.00000 & 0.00000 \\
\hline ne & 0.00000 & 0.00000 & 0.00000 & 0.00000 & 0.00 & 0.00 & 200 & & 000 & & 0000 & 0.00000 & & 0.00000 & 00 & 0.00000 & 0.00000 \\
\hline thanol & 0.01000 & 0.19000 & 0.20000 & & & & 000 & & & & 0.00000 & 0.00000 & 0.00010 & 0.01000 & 0.00010 & 0.00990 & 0.01000 \\
\hline Phosphoric & & & & & & & & & & & & & & 0.00000 & & 0000 & 0.00000 \\
\hline 13 & 0.00000 & 0.00000 & 0.00000 & 0.000 & 100 & 0.01225 & & 0122 & 0.00122 & 00122 & 0.01102 & 0.01102 & 0.00000 & 0.00000 & 0.00000 & 0.00000 & .00000 \\
\hline
\end{tabular}


Table S2 Summary of life cycle impact assessment methods used in this work

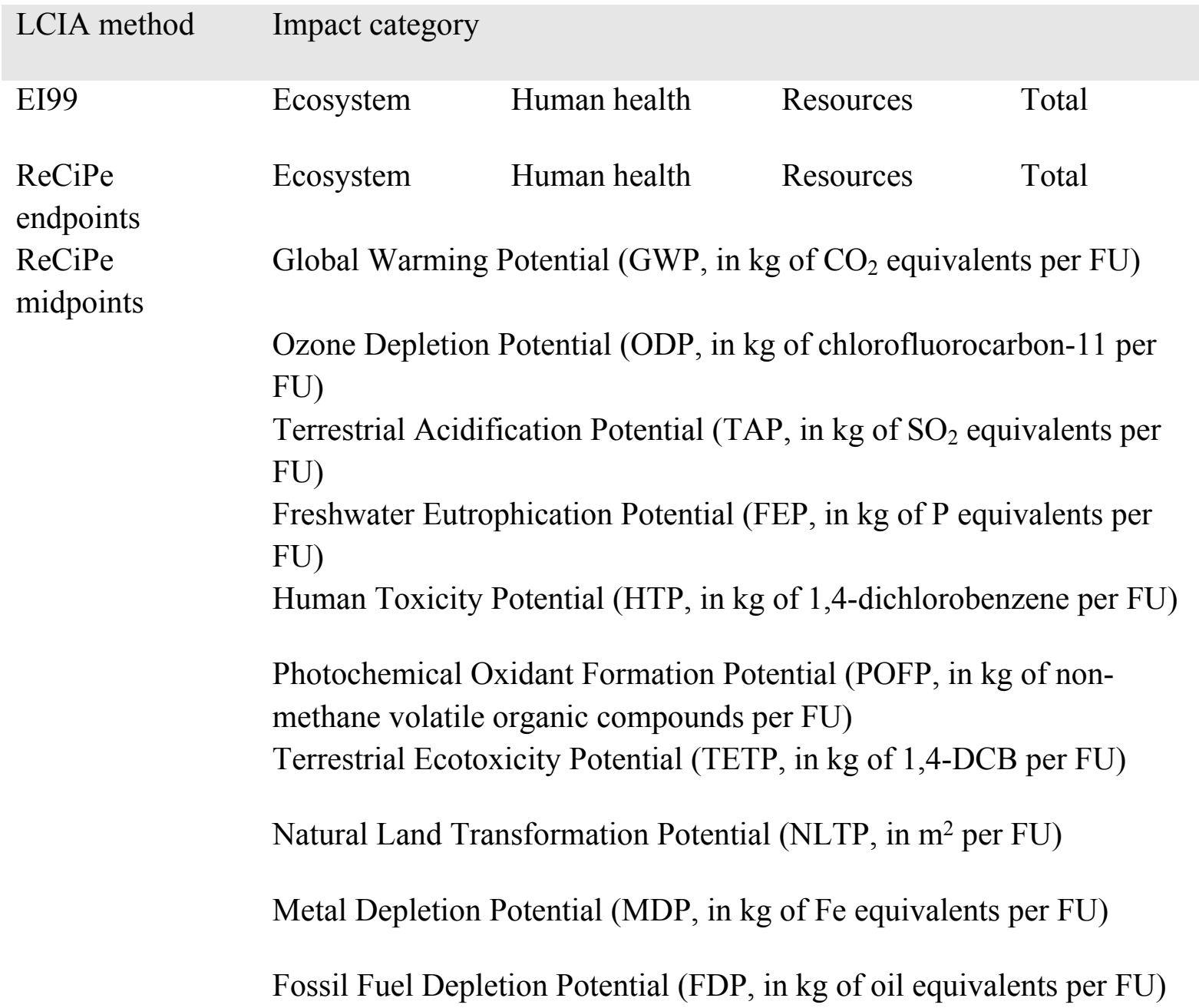


Table S3 Methods to complete data gaps of LCI in the life cycle assessment

\begin{tabular}{|c|c|c|}
\hline Data types of missing LCI & The solutions to deal with data gaps & Data sources/References \\
\hline \multirow{3}{*}{ Chemical materials } & Retrosynthetic breakdown method & https://www.reaxys.com/ \\
\hline & $\begin{array}{l}\text { Substitution method with the LCI data of } \\
\text { the material with similar structure }\end{array}$ & \multirow[t]{2}{*}{3} \\
\hline & Substitution method with generic data & \\
\hline Energy consumption & $\begin{array}{l}\text { Process energy in organic chemical } \\
\text { manufacturing was estimated with } 4 \mathrm{MJ} \mathrm{kg}^{-} \\
1\end{array}$ & 3,4 \\
\hline Infrastructures & $\begin{array}{l}\text { Generic data "chemical factory } \\
\text { construction, organics, RoW" in Ecoinvent } \\
\text { database is used to model the LCI of all } \\
\text { infrastructure }\end{array}$ & https://www.ecoinvent.org/ \\
\hline LCI of wastes treatment & $\begin{array}{l}\text { treatment of spent solvent mixture, } \\
\text { hazardous waste incineration, RoW" in } \\
\text { Ecoinvent database }\end{array}$ & https://www.ecoinvent.org/ \\
\hline
\end{tabular}


Table S4 Life-cycle inventory data of chemicals used to build ML predictive models in the study

\begin{tabular}{|c|c|c|c|c|c|c|c|c|c|}
\hline No. & Chemical & $\begin{array}{c}\text { EI99 - } \\
\text { Ecosystem }\end{array}$ & $\begin{array}{l}\text { EI99 - } \\
\text { Human } \\
\text { health }\end{array}$ & $\begin{array}{l}\text { EI99 - } \\
\text { Resources }\end{array}$ & $\begin{array}{l}\text { EI99 - } \\
\text { Total }\end{array}$ & $\begin{array}{l}\text { ReCiPe - } \\
\text { Ecosystem }\end{array}$ & $\begin{array}{c}\text { ReCiPe - } \\
\text { Human } \\
\text { health }\end{array}$ & $\begin{array}{l}\text { ReCiPe - } \\
\text { Resources }\end{array}$ & $\begin{array}{l}\text { ReCiPe - } \\
\text { Total }\end{array}$ \\
\hline 1 & 1,1-difluoroethane, HFC-152a & 0.030916965 & 0.22819244 & 0.10240473 & 0.36151413 & 0.10571136 & 0.24970611 & 0.16454156 & 0.519959 \\
\hline 2 & 1-propanol & 0.020516276 & 0.17294621 & 0.23305292 & 0.4265154 & 0.092209619 & 0.20137072 & 0.27514023 & 0.568721 \\
\hline 3 & 2,4-dichlorophenol & 0.021370055 & 0.17728879 & 0.17371007 & 0.37236892 & 0.079539914 & 0.19146349 & 0.22934708 & 0.50035 \\
\hline 4 & 2,4-dichlorotoluene & 0.015238676 & 0.11952884 & 0.15699566 & 0.29176317 & 0.058422759 & 0.13817589 & 0.18190742 & 0.378506 \\
\hline 5 & 2,4-dinitrotoluene & 0.023303968 & 0.093422143 & 0.15338025 & 0.27010636 & 0.042908317 & 0.1080529 & 0.15608575 & 0.307047 \\
\hline 6 & 2,4-di-tert-butylphenol & 0.012539884 & 0.11302558 & 0.21990279 & 0.34546826 & 0.063466009 & 0.13704526 & 0.23970869 & 0.44022 \\
\hline 7 & $\begin{array}{l}\text { 2,5-dimethylhexane-2,5- } \\
\text { dihydroperoxide }\end{array}$ & 0.022598827 & 0.17988617 & 0.2197974 & 0.42228239 & 0.091742007 & 0.20796649 & 0.25695107 & 0.55666 \\
\hline 8 & 2,6-di-tert-butylphenol & 0.012937532 & 0.11693938 & 0.22934134 & 0.35921825 & 0.065877239 & 0.14213826 & 0.24968848 & 0.457704 \\
\hline 9 & 2-butanol & 0.012157335 & 0.10725725 & 0.26507904 & 0.38449362 & 0.08340452 & 0.16860865 & 0.27524714 & 0.52726 \\
\hline 10 & 2-methyl-2-butanol & 0.0126386 & 0.09139078 & 0.21286207 & 0.31689145 & 0.06595344 & 0.13147502 & 0.22783984 & 0.425268 \\
\hline 11 & 2-nitroaniline & 0.033110601 & 0.24514331 & 0.24509504 & 0.52334895 & 0.12527572 & 0.28040438 & 0.31175567 & 0.717436 \\
\hline 12 & 2-pyridinol & 0.4817093 & 3.2638108 & 5.7675872 & 9.5131073 & 1.9432057 & 4.1858117 & 6.0948899 & 12.22391 \\
\hline 13 & 3-methyl-1-butyl acetate & 0.022665561 & 0.16920582 & 0.27069638 & 0.46256776 & 0.11060625 & 0.22415153 & 0.30870528 & 0.643463 \\
\hline 14 & 4-methyl-2-pentanone & 0.015575556 & 0.12506446 & 0.30725221 & 0.44789222 & 0.081966723 & 0.17841787 & 0.31143153 & 0.571816 \\
\hline 15 & 4-tert-butylbenzaldehyde & 0.016756364 & 0.12417031 & 0.28080638 & 0.42173305 & 0.084668329 & 0.1753483 & 0.28831007 & 0.548327 \\
\hline 16 & 4-tert-butyltoluene & 0.007230874 & 0.052985227 & 0.22767041 & 0.28788651 & 0.043661847 & 0.088028836 & 0.21519066 & 0.346881 \\
\hline 17 & acetaldehyde & 0.007685992 & 0.056748097 & 0.15527807 & 0.21971216 & 0.034449096 & 0.074446813 & 0.15591873 & 0.264815 \\
\hline 18 & acetanilide & 0.030641565 & 0.20820872 & 0.2529873 & 0.49183758 & 0.10235413 & 0.24075778 & 0.29987913 & 0.642991 \\
\hline 19 & acetic acid & 0.009068841 & 0.069793556 & 0.13290221 & 0.2117646 & 0.034642938 & 0.075051765 & 0.14168488 & 0.25138 \\
\hline 20 & acetic anhydride & 0.017428197 & 0.1301163 & 0.1935067 & 0.3410512 & 0.074304688 & 0.15642152 & 0.21797324 & 0.448699 \\
\hline 21 & acetoacetic acid & 0.034315585 & 0.24593499 & 0.405438 & 0.68568857 & 0.16266721 & 0.32878296 & 0.44096972 & 0.93242 \\
\hline 22 & acetone cyanohydrin & 0.007232666 & 0.068532249 & 0.25945208 & 0.335217 & 0.070007689 & 0.14749945 & 0.23983446 & 0.457342 \\
\hline 23 & acetone & 0.007465376 & 0.060793698 & 0.19790287 & 0.26616194 & 0.043880684 & 0.094507345 & 0.19215925 & 0.330547 \\
\hline 24 & acetyl chloride & 0.031620738 & 0.23739095 & 0.29302277 & 0.56203446 & 0.14297308 & 0.29820603 & 0.34216517 & 0.783344 \\
\hline
\end{tabular}




\begin{tabular}{|c|c|c|c|c|c|c|c|c|c|}
\hline 25 & acetylene & 0.02291506 & 0.31274718 & 0.095003115 & 0.43066535 & 0.11618009 & 0.29137042 & 0.21052766 & 0.618078 \\
\hline 26 & aclonifen & 0.045775417 & 0.34782829 & 0.29027558 & 0.68387928 & 0.16385056 & 0.37536252 & 0.39739239 & 0.936605 \\
\hline 27 & acrolein & 0.007414167 & 0.069805477 & 0.1913372 & 0.26855685 & 0.044602551 & 0.095155758 & 0.19289166 & 0.33265 \\
\hline 28 & acrylic acid & 0.004868275 & 0.046502088 & 0.14520378 & 0.19657414 & 0.03895874 & 0.076714199 & 0.1406721 & 0.256345 \\
\hline 29 & adipic acid & 0.024604064 & 0.23449856 & 0.22750893 & 0.48661156 & 0.27093715 & 0.48913035 & 0.28488589 & 1.044953 \\
\hline 30 & alkylbenzene sulfonate & 0.012019711 & 0.07224502 & 0.16107173 & 0.24533647 & 0.033655727 & 0.08121403 & 0.17272137 & 0.287591 \\
\hline 31 & alkylbenzene & 0.011515829 & 0.079259347 & 0.20061146 & 0.29138663 & 0.040935511 & 0.092805556 & 0.21326349 & 0.347005 \\
\hline 32 & allyl chloride & 0.006650541 & 0.059846251 & 0.099080981 & 0.16557777 & 0.029062823 & 0.066614021 & 0.1080954 & 0.203772 \\
\hline 33 & alpha-naphthol & 0.023353084 & 0.15084997 & 0.24303936 & 0.41724242 & 0.06646991 & 0.15465923 & 0.26065213 & 0.481781 \\
\hline 34 & alpha-picoline & 0.017956135 & 0.12188628 & 0.2722767 & 0.41211912 & 0.07293674 & 0.15736658 & 0.27907899 & 0.509382 \\
\hline 35 & aniline & 0.031759771 & 0.21691252 & 0.24251123 & 0.49118353 & 0.10224804 & 0.24942309 & 0.29381374 & 0.645485 \\
\hline 36 & anthranilic acid & 0.033458778 & 0.20774689 & 0.29365845 & 0.53486412 & 0.16299472 & 0.32655392 & 0.30311857 & 0.792667 \\
\hline 37 & anthraquinone & 0.65636833 & 0.97602261 & 0.72330741 & 2.3556984 & 0.4071317 & 0.98831188 & 1.8102664 & 3.20571 \\
\hline 38 & ascorbic acid & 0.16897627 & 0.18403905 & 0.095231707 & 0.44824703 & 0.14949989 & 0.16988023 & 0.13750747 & 0.456888 \\
\hline 39 & atrazine & 0.042897423 & 0.31689172 & 0.33828247 & 0.69807162 & 0.15979631 & 0.36471195 & 0.40473 & 0.929238 \\
\hline 40 & azodicarbonamide & 0.10632506 & 0.7391683 & 0.47633635 & 1.3218297 & 0.29208675 & 0.71965551 & 0.65155859 & 1.663301 \\
\hline 41 & benzal chloride & 0.012041858 & 0.098001945 & 0.15240088 & 0.26244468 & 0.05168197 & 0.12008348 & 0.1689384 & 0.340704 \\
\hline 42 & benzaldehyde & 0.023361182 & 0.18261079 & 0.25761664 & 0.46358861 & 0.097647599 & 0.22368501 & 0.29210006 & 0.613433 \\
\hline 43 & benzene & 0.008705678 & 0.079753571 & 0.17403731 & 0.26249655 & 0.035703379 & 0.087948358 & 0.20935333 & 0.333005 \\
\hline 44 & benzyl alcohol & 0.021541683 & 0.14109171 & 0.23091888 & 0.39355228 & 0.082523247 & 0.17977243 & 0.2523714 & 0.514667 \\
\hline 45 & benzyl chloride & 0.009888401 & 0.054334185 & 0.17573105 & 0.23995363 & 0.043824282 & 0.089304265 & 0.17426482 & 0.307393 \\
\hline 46 & bisphenol A & 0.016999522 & 0.14056944 & 0.24687018 & 0.40443914 & 0.076192587 & 0.16976854 & 0.27943971 & 0.525401 \\
\hline 47 & boric oxide & 0.032497231 & 0.24748155 & 0.10206366 & 0.38204244 & 0.050807276 & 0.17755727 & 0.12853289 & 0.356897 \\
\hline 48 & boron carbide & 0.10804777 & 0.98249128 & 0.35925218 & 1.4497912 & 0.33597925 & 0.89102259 & 0.55815226 & 1.785154 \\
\hline 49 & boron trifluoride & 0.041857554 & 0.26335425 & 0.13475081 & 0.43996262 & 0.058741429 & 0.20723093 & 0.16600172 & 0.431974 \\
\hline 50 & butadiene & 0.001346313 & 0.018128576 & 0.20806127 & 0.22753616 & 0.020537563 & 0.040693142 & 0.185702 & 0.246933 \\
\hline 51 & butane & 0.00305291 & 0.025381275 & 0.13319786 & 0.16163204 & 0.016374605 & 0.03374478 & 0.12818723 & 0.178307 \\
\hline 52 & butane-1,4-diol & 0.021299407 & 0.17871083 & 0.1874879 & 0.38749814 & 0.098291794 & 0.2111235 & 0.2390254 & 0.548441 \\
\hline 53 & butyl acetate & 0.017192464 & 0.12915303 & 0.23319237 & 0.37953786 & 0.07504165 & 0.1584679 & 0.2540806 & 0.48759 \\
\hline 54 & butyl acrylate & 0.016701426 & 0.13386792 & 0.2367661 & 0.38733544 & 0.084848052 & 0.18072975 & 0.2581295 & 0.523707 \\
\hline 55 & butyldiglycol acetate & 0.013675768 & 0.1220048 & 0.2227697 & 0.35845027 & 0.062455581 & 0.13439413 & 0.23568513 & 0.432535 \\
\hline
\end{tabular}




\begin{tabular}{|c|c|c|c|c|c|c|c|c|c|}
\hline 56 & captan & 0.021206949 & 0.15343452 & 0.20450872 & 0.37915019 & 0.079910727 & 0.17702561 & 0.2370118 & 0.493948 \\
\hline 57 & carbon disulfide & 0.004969812 & 0.032255693 & 0.13280906 & 0.17003456 & 0.016421099 & 0.035316786 & 0.1275515 & 0.179289 \\
\hline 58 & carbon tetrachloride & 0.005263484 & 0.056451607 & 0.063153537 & 0.12486863 & 0.02936159 & 0.080473018 & 0.06271145 & 0.172546 \\
\hline 59 & chlorine & 0.005763569 & 0.05842171 & 0.018253579 & 0.082438859 & 0.021086348 & 0.053134764 & 0.038533114 & 0.112754 \\
\hline 60 & chloroacetic acid & 0.013690099 & 0.10921377 & 0.10998426 & 0.23288812 & 0.046353963 & 0.11158094 & 0.13652287 & 0.294458 \\
\hline 61 & chloroacetyl chloride & 0.029898391 & 0.22822688 & 0.17042948 & 0.42855475 & 0.084072376 & 0.23702559 & 0.22547467 & 0.546573 \\
\hline 62 & chlorodifluoromethane & 0.034884816 & 0.65479161 & 0.17641511 & 0.86609153 & 1.3209287 & 2.457265 & 0.2315521 & 4.009746 \\
\hline 63 & chloromethyl methyl ether & 0.009951476 & 0.075195682 & 0.11544144 & 0.20058859 & 0.032805151 & 0.078156718 & 0.12583685 & 0.236799 \\
\hline 64 & chloronitrobenzene & 0.021526319 & 0.16070893 & 0.17399447 & 0.35622973 & 0.083340525 & 0.18594176 & 0.21810267 & 0.487385 \\
\hline 65 & chloropropionic acid & 0.017316192 & 0.13906799 & 0.1468269 & 0.30321108 & 0.064091168 & 0.15074869 & 0.18131819 & 0.396158 \\
\hline 66 & chlorosulfonic acid & 0.014692526 & 0.085795843 & 0.037497171 & 0.13798554 & 0.018907123 & 0.065065527 & 0.052844345 & 0.136817 \\
\hline 67 & chlorothalonil & 0.023593626 & 0.15861267 & 0.1652028 & 0.3474091 & 0.078148774 & 0.18263675 & 0.20344369 & 0.464229 \\
\hline 68 & chlorotoluron & 0.037670892 & 0.27122691 & 0.25470264 & 0.56360044 & 0.13788214 & 0.31771754 & 0.3168553 & 0.772455 \\
\hline 69 & citric acid & 0.14576222 & 0.25673709 & 0.11507552 & 0.51757483 & 0.62824255 & 0.29299911 & 0.20194511 & 1.123187 \\
\hline 70 & cumene & 0.010047726 & 0.086028393 & 0.20917214 & 0.30524826 & 0.045189886 & 0.10328356 & 0.23174832 & 0.380222 \\
\hline 71 & cyanoacetic acid & 0.026060143 & 0.19928319 & 0.23591687 & 0.46126021 & 0.098019529 & 0.22277787 & 0.27528117 & 0.596079 \\
\hline 72 & cyanogen chloride & 0.020888211 & 0.16907425 & 0.18309038 & 0.37305284 & 0.091915571 & 0.21014765 & 0.21976602 & 0.521829 \\
\hline 73 & cyanuric chloride & 0.02415092 & 0.19906422 & 0.19586937 & 0.41908451 & & 0.23748643 & 0.24107183 & 0.581422 \\
\hline 74 & cyclohexane & 0.012416292 & 0.1021941 & 0.1920219 & 0.30663229 & 0.047593369 & 0.11329239 & 0.23142393 & 0.39231 \\
\hline 75 & cyclohexanol & 0.013717655 & 0.11521262 & 0.1958358 & 0.32476607 & 0.054291649 & 0.12687234 & 0.23143141 & 0.412595 \\
\hline 76 & cyclohexanone & 0.02149676 & 0.16548146 & 0.22567255 & 0.41265077 & 0.086926006 & 0.19653457 & 0.28731404 & 0.570775 \\
\hline 77 & decabromodiphenyl ether & 0.062827351 & 0.44610198 & 0.42922558 & 0.93815492 & 0.24613754 & 0.56761295 & 0.51241808 & 1.326169 \\
\hline 78 & dichloromethane & 0.008112384 & 0.11520053 & 0.081258217 & 0.20457113 & 0.060702087 & 0.16403341 & 0.088108238 & 0.312844 \\
\hline 79 & dichloropropene & 0.016512254 & 0.13069609 & 0.26310653 & 0.41031488 & 0.071605586 & 0.15833498 & 0.28022114 & 0.510162 \\
\hline 80 & diethanolamine & 0.011148308 & 0.11000414 & 0.19707702 & 0.31822947 & 0.052841203 & 0.11314903 & 0.20113378 & 0.367124 \\
\hline 81 & diethyl ether & 0.060952039 & 0.42307757 & 0.34661319 & 0.83064279 & 0.14026973 & 0.37995268 & 0.4263029 & 0.946525 \\
\hline 82 & diethylene glycol & 0.008637233 & 0.079103003 & 0.16120357 & 0.24894381 & 0.041825097 & 0.093186619 & 0.16860652 & 0.303618 \\
\hline 83 & dimethyl carbonate & 0.011067301 & 0.078862529 & 0.14623536 & 0.23616519 & 0.042216748 & 0.09115912 & 0.1544471 & 0.287823 \\
\hline 84 & dimethyl ether & 0.005995542 & 0.039768925 & 0.17075313 & 0.21651759 & 0.025907642 & 0.051993234 & 0.16279704 & 0.240698 \\
\hline 85 & dimethyl malonate & 0.031237965 & 0.22435656 & 0.26574012 & 0.52133465 & 0.1098453 & 0.24851208 & 0.30978485 & 0.668142 \\
\hline 86 & dimethyl sulfate & 0.014976803 & 0.097921462 & 0.10050968 & 0.21340795 & 0.028118919 & 0.079783932 & 0.11139679 & 0.2193 \\
\hline
\end{tabular}




\begin{tabular}{|c|c|c|c|c|c|c|c|c|c|}
\hline 87 & dimethyl sulfide & 0.009079304 & 0.062584076 & 0.20938588 & 0.28104926 & 0.035648038 & 0.075107531 & 0.20450332 & 0.315259 \\
\hline 88 & dimethyl sulfoxide & 0.007678552 & 0.052490887 & 0.17006816 & 0.2302376 & 0.02852925 & 0.060545195 & 0.16635312 & 0.255428 \\
\hline 89 & dimethylacetamide & 0.016863151 & 0.1190255 & 0.23508818 & 0.37097683 & 0.063364605 & 0.13605546 & 0.24499036 & 0.44441 \\
\hline 90 & dimethylamine & 0.01187143 & 0.075684717 & 0.2276539 & 0.31521005 & 0.047427702 & 0.098520044 & 0.21998669 & 0.365934 \\
\hline 91 & dimethylaminopropylamine & 0.017982715 & 0.12013282 & 0.27431169 & 0.41242722 & 0.072883994 & 0.15536805 & 0.27540806 & 0.50366 \\
\hline 92 & dinitrogen tetroxide & 0.035023019 & 0.10942879 & 0.073870456 & 0.21832226 & 0.03404726 & 0.10725903 & 0.083912248 & 0.225219 \\
\hline 93 & dioxane & 0.019432964 & 0.17213293 & 0.25522105 & 0.44678694 & 0.095136182 & 0.20821783 & 0.28818961 & 0.591544 \\
\hline 94 & dipropyl amine & 0.025710953 & 0.1902466 & 0.3058689 & 0.52182645 & 0.11497019 & 0.24050487 & 0.34448392 & 0.699959 \\
\hline 95 & $\begin{array}{l}\text { dipropylene glycol monomethyl } \\
\text { ether }\end{array}$ & 0.022983579 & 0.21303791 & 0.2268957 & 0.46291718 & 0.093229774 & 0.2253034 & 0.27593307 & 0.594466 \\
\hline 96 & dodecanol & 0.59062034 & 0.25136727 & 0.14086168 & 0.9828493 & 1.6125584 & 0.20139912 & 0.17061382 & 1.984571 \\
\hline 97 & Diethylenetriaminepentaacetic acid & 0.019010568 & 0.14212107 & 0.20467976 & 0.3658114 & 0.075918214 & 0.16890656 & 0.22453739 & 0.469362 \\
\hline 98 & Ethylenediaminetetraacetic acid & 0.019064873 & 0.14184668 & 0.20775962 & 0.36867118 & 0.07789447 & 0.17186207 & 0.22769348 & 0.47745 \\
\hline 99 & epichlorohydrin & 0.013375479 & 0.10227791 & 0.10306603 & 0.21871942 & 0.055140989 & 0.11899021 & 0.1262044 & 0.300336 \\
\hline 100 & ethane & 0.003042548 & 0.025303544 & 0.13321374 & 0.16155983 & 0.01629074 & 0.033589486 & 0.12817669 & 0.178057 \\
\hline 101 & ethanol & 0.10254151 & 0.088447472 & 0.032221995 & 0.22321098 & 0.3416203 & 0.060566106 & 0.039384859 & 0.441571 \\
\hline 102 & ethyl a & 0.013417298 & 0.092875604 & 0.19440927 & 0.30070217 & 0.054334677 & 0.11349935 & 0.20393752 & 0.371772 \\
\hline 103 & ethyl benzene & 0.01061743 & 0.092130603 & 0.20031902 & 0.30306705 & 0.044508972 & 0.10461566 & 0.228434 & 0.377559 \\
\hline 104 & ethyl tert-butyl ether & 0.14271403 & 0.1185779 & 0.12522933 & 0.38652126 & 0.2546382 & 0.068571771 & 0.12139709 & 0.444607 \\
\hline 105 & ethylamine & 0.013958958 & 0.082549076 & 0.22242453 & 0.31893256 & 0.057227749 & & 0.21773122 & 0.392943 \\
\hline 106 & ethylene bromide & 0.022474656 & 0.16935491 & 0.23055116 & 0.42238073 & 0.11687151 & 0.24460364 & 0.26640021 & 0.627875 \\
\hline 107 & ethylene carbonate & 0.007330267 & 0.051221737 & 0.10295184 & 0.16150385 & 0.029624642 & 0.062758129 & 0.10709465 & 0.199477 \\
\hline 108 & ethylene dichloride & 0.009585182 & 0.058825762 & 0.076858365 & 0.14526931 & 0.025460029 & 0.059990833 & 0.087967777 & 0.173419 \\
\hline 109 & ethylene glycol diethyl ether & 0.01773048 & 0.13624576 & 0.2018103 & 0.35578654 & 0.066065705 & 0.14677433 & 0.22183501 & 0.434675 \\
\hline 110 & ethylene glycol dimethyl ether & 0.009933506 & 0.087586216 & 0.1982287 & 0.29574842 & 0.045023624 & 0.096023287 & 0.20002527 & 0.341072 \\
\hline 111 & ethylene glycol monoethyl ether & 0.009061482 & 0.08676858 & 0.18104247 & 0.27687253 & 0.04456382 & 0.09663894 & 0.1840906 & 0.325293 \\
\hline 112 & ethylene glycol & 0.007926783 & 0.07142407 & 0.13901519 & 0.21836604 & 0.036999819 & 0.082855012 & 0.14652957 & 0.266384 \\
\hline 113 & ethylene oxide & 0.005934028 & 0.066401348 & 0.18481424 & 0.25714962 & 0.04065456 & 0.087654444 & 0.18453832 & 0.312847 \\
\hline 114 & ethylenediamine & 0.027887915 & 0.19981926 & 0.29193231 & 0.51963949 & 0.10045552 & 0.22946069 & 0.31923443 & 0.649151 \\
\hline 115 & folpet & 0.024014449 & 0.1521966 & 0.20597308 & 0.38218412 & 0.075696104 & 0.17395245 & 0.23405525 & 0.483704 \\
\hline 116 & formaldehyde & 0.004867558 & 0.034036905 & 0.11836346 & 0.15726792 & 0.019439912 & 0.040654459 & 0.11369179 & 0.173786 \\
\hline
\end{tabular}




\begin{tabular}{|c|c|c|c|c|c|c|c|c|c|}
\hline 117 & formic acid & 0.01384111 & 0.12094571 & 0.13385108 & 0.2686379 & 0.063564823 & 0.13865657 & 0.16654071 & 0.368762 \\
\hline 118 & glucose & 0.14838689 & 0.082916062 & 0.037634419 & 0.26893737 & 0.10089461 & 0.067833997 & 0.051530396 & 0.220259 \\
\hline 119 & glycerine & 0.39060654 & 0.11558016 & 0.052255134 & 0.55844183 & 1.1494732 & 0.14705175 & 0.063720059 & 1.360245 \\
\hline 120 & glycine & 0.029173077 & 0.21013997 & 0.20165133 & 0.44096437 & 0.09625005 & 0.2227156 & 0.2526494 & 0.571615 \\
\hline 121 & glyoxal & 0.011142916 & 0.13738926 & 0.17413708 & 0.32266926 & 0.058562621 & 0.13276805 & 0.19471917 & 0.38605 \\
\hline 122 & glyphosate & 0.067488787 & 0.53857776 & 0.33449897 & 0.94056551 & 0.23446582 & 0.65311503 & 0.50265259 & 1.390233 \\
\hline 123 & hydrazine sulfate & 0.041413414 & 0.30266533 & 0.15257925 & 0.496658 & 0.11441408 & 0.28705082 & 0.23897952 & 0.640444 \\
\hline 124 & hydrazine & 0.086775049 & 0.67895577 & 0.36713613 & 1.1328669 & 0.27425917 & 0.66660325 & 0.56383609 & 1.504699 \\
\hline 125 & hydrogen cyanide & 0.010924564 & 0.075703251 & 0.27687746 & 0.36350528 & 0.077153042 & 0.1506901 & 0.25818807 & 0.486031 \\
\hline 126 & hydrogen peroxide & 0.017137456 & 0.058276126 & 0.051122013 & 0.12653559 & 0.028535667 & 0.066031702 & 0.065195241 & 0.159763 \\
\hline 127 & hydroquinone & 0.020194994 & 0.14131241 & 0.21828011 & 0.37978751 & 0.071093598 & 0.16164051 & 0.25680553 & 0.48954 \\
\hline 128 & hydroxylamine & 0.11746024 & 0.93632564 & 0.73293082 & 1.7867167 & 0.316028 & 1.1041538 & 0.84986749 & 2.270049 \\
\hline 129 & imidazole & 0.0226075 & 0.17778437 & 0.29001709 & 0.49040895 & 0.092030014 & 0.20028653 & 0.30550933 & 0.597826 \\
\hline 130 & isobutanol & 0.011492922 & 0.10367443 & 0.21985364 & 0.335021 & 0.059669867 & 0.12897467 & 0.23246317 & 0.421108 \\
\hline 131 & isobutyl acetate & 0.017569216 & 0.12901519 & 0.24450741 & 0.39109182 & 0.077135089 & 0.16013698 & 0.26370442 & 0.500976 \\
\hline 132 & isohexane & 0.008357052 & 0.041401529 & 0.14656005 & 0.19631863 & 0.023545896 & 0.045496013 & 0.14044674 & 0.209489 \\
\hline 133 & isopropanol & 0.007026195 & 0.054462561 & 0.18924454 & 0.2507333 & 0.03867713 & 0.080671989 & 0.18112647 & 0.300476 \\
\hline 134 & isopropyl acetate & 0.020819413 & 0.15925675 & 0.25281306 & 0.43288922 & 0.09050918 & 0.19372761 & 0.28206885 & 0.566306 \\
\hline 135 & isopropylamine & 0.015519383 & 0.10158325 & 0.2671667 & 0.38426934 & 0.070315837 & 0.14625186 & 0.26240011 & 0.478968 \\
\hline 136 & lactic acid & 0.018921779 & 0.13508169 & 0.21969052 & 0.37369399 & 0.080585298 & 0.17542429 & 0.23682533 & 0.492835 \\
\hline 137 & maleic anhydride & 0.010272623 & 0.081262082 & 0.18245716 & 0.27399186 & 0.058088639 & 0.11442484 & 0.1947172 & 0.367231 \\
\hline 138 & melamine & 0.038057579 & 0.20326738 & 0.26966804 & 0.510993 & 0.10127555 & 0.22988331 & 0.27520557 & 0.606364 \\
\hline 139 & methacrylic acid & 0.032937218 & 0.21744194 & 0.34875456 & 0.59913372 & 0.12143389 & 0.27528255 & 0.36849916 & 0.765216 \\
\hline 140 & methane sulfonic acid & 0.01522912 & 0.086408177 & 0.048001286 & 0.14963858 & 0.022304578 & 0.071374893 & 0.062078527 & 0.155758 \\
\hline 141 & methanol & 0.001903617 & 0.016360267 & 0.10931585 & 0.12757974 & 0.012545088 & 0.024237535 & 0.10064109 & 0.137424 \\
\hline 142 & methyl acrylate & 0.007907861 & 0.061166566 & 0.19497248 & 0.26404691 & 0.050147013 & 0.10650769 & 0.18782207 & 0.344477 \\
\hline 143 & bromopropane & 0.019815926 & 0.13751897 & 0.21998399 & 0.37731889 & 0.095199572 & 0.19647178 & 0.24408118 & 0.535753 \\
\hline 144 & methyl ethyl ketone & 0.005919344 & 0.049194053 & 0.17719687 & 0.23231027 & 0.034726399 & 0.071120551 & 0.17072393 & 0.276571 \\
\hline 145 & methyl formate & 0.013306966 & 0.10982408 & 0.21074095 & 0.333872 & 0.056376022 & 0.12205658 & 0.2275208 & 0.405953 \\
\hline 146 & methyl iodide & 0.025450944 & 0.19085345 & 0.2280872 & 0.4443916 & 0.12947039 & 0.26173493 & 0.27487209 & 0.666077 \\
\hline 147 & methyl tert-butyl ether & 0.004181917 & 0.029156688 & 0.18014626 & 0.21348487 & 0.020930051 & 0.043378219 & 0.16482366 & 0.229132 \\
\hline
\end{tabular}




\begin{tabular}{|c|c|c|c|c|c|c|c|c|c|}
\hline 148 & methyl-3-methoxypropionate & 0.010192263 & 0.076194186 & 0.19319688 & 0.27958333 & 0.052728121 & 0.11299872 & 0.19192551 & 0.357652 \\
\hline 149 & methylamine & 0.013720558 & 0.08075275 & 0.21043548 & 0.30490879 & 0.051520988 & 0.10732155 & 0.205444 & 0.364287 \\
\hline 150 & methylchloride & 0.007954146 & 0.11084127 & 0.11726142 & 0.23605683 & 0.054844445 & 0.14792786 & 0.12032237 & 0.323095 \\
\hline 151 & methylcyclohexane & 0.013527218 & 0.093678558 & 0.26992827 & 0.37713404 & 0.071749271 & 0.14281342 & 0.27295881 & 0.487522 \\
\hline 152 & N-methyl-2-pyrrolidone & 0.041068045 & 0.30056832 & 0.41236246 & 0.75399882 & 0.16587917 & 0.35643363 & 0.47147528 & 0.993788 \\
\hline 153 & $\mathrm{~N}, \mathrm{~N}$-dimethylformamide & 0.015241727 & 0.1021134 & 0.21449719 & 0.33185232 & 0.055874301 & 0.11898063 & 0.22185683 & 0.396712 \\
\hline 154 & naphthalene sulfonic acid & 0.012950683 & 0.11178818 & 0.039741708 & 0.16448057 & 0.028899688 & 0.08677057 & 0.10230175 & 0.217972 \\
\hline 155 & nitrobenzene & 0.020664083 & 0.13756965 & 0.15958552 & 0.31781926 & 0.065910946 & 0.15654999 & 0.19217059 & 0.414632 \\
\hline 156 & o-aminophenol & 0.02772692 & 0.19786765 & 0.26551926 & 0.49111383 & 0.11571366 & 0.24969281 & 0.30754581 & 0.672952 \\
\hline 157 & o-chlorobenzaldehyde & 0.041207205 & 0.32736993 & 0.27028421 & 0.63886135 & 0.16151758 & 0.36727341 & 0.35861196 & 0.887403 \\
\hline 158 & o-chlorotoluene & 0.012733335 & 0.1016958 & 0.18077833 & 0.29520746 & 0.053505616 & 0.1228353 & 0.19410002 & 0.370441 \\
\hline 159 & o-cresol & 0.01704465 & 0.13801266 & 0.24963432 & 0.40469163 & 0.07230476 & 0.16177486 & 0.28342581 & 0.517505 \\
\hline 160 & o-nitrophenol & 0.017577407 & 0.12643816 & 0.17666528 & 0.32068084 & 0.074542831 & 0.16091556 & 0.20307525 & 0.438534 \\
\hline 161 & p-chlorophenol & 0.02019077 & 0.16651256 & 0.19917618 & 0.38587951 & 0.078053786 & 0.18388246 & 0.24930601 & 0.511242 \\
\hline 162 & p-nitrophenol & 0.017527348 & 0.12571759 & 0.17666899 & 0.31991393 & 0.074357209 & 0.16035661 & 0.20290032 & 0.437614 \\
\hline 163 & p-nitrotoluene & 0.012332648 & 0.072603878 & 0.18906975 & 0.27400627 & 0.065460171 & 0.12824346 & 0.1812081 & 0.374912 \\
\hline 164 & pentaerythritol & 0.01233179 & 0.09041766 & 0.15806256 & 0.26081201 & 0.046898892 & 0.10316447 & 0.1698042 & 0.319868 \\
\hline 165 & pentane & 0.002141093 & 0.022247846 & 0.20376928 & 0.22815822 & 0.017704485 & 0.036552716 & 0.18606423 & 0.240321 \\
\hline 166 & perfluoropentane & 0.040460437 & 0.38639568 & 0.24482316 & 0.67167927 & 0.29164842 & 0.63414348 & 0.30781355 & 1.233606 \\
\hline 167 & phenol & 0.013399273 & 0.11442411 & 0.21588444 & 0.34370783 & 0.058471688 & 0.13309298 & 0.24724923 & 0.438814 \\
\hline 168 & phenyl acetic acid & 0.024680233 & 0.17576694 & 0.28902316 & 0.48947033 & 0.10693716 & 0.23098197 & 0.31362381 & 0.651543 \\
\hline 169 & phenyl isocyanate & 0.044002404 & 0.31940114 & 0.28798592 & 0.65138946 & 0.14518154 & 0.36445486 & 0.3749796 & 0.884616 \\
\hline 170 & phosgene, liquid & 0.010775097 & 0.1017545 & 0.069667539 & 0.18219714 & 0.039124865 & 0.1139368 & 0.099307484 & 0.252369 \\
\hline 171 & phosphorous chloride & 0.02714003 & 0.23412523 & 0.071927782 & 0.33319304 & 0.083976088 & 0.32803591 & 0.15911308 & 0.571125 \\
\hline 172 & phosphorus pentachloride & 0.02338932 & 0.16804812 & 0.053233629 & 0.24467107 & 0.066264172 & 0.30238125 & 0.11760122 & 0.486247 \\
\hline 173 & phosphoryl chloride & 0.029769743 & 0.25522914 & 0.078678723 & 0.3636776 & 0.094146394 & 0.33885849 & 0.17204098 & 0.605046 \\
\hline 174 & phthalic anhydride & 0.015823899 & 0.072668709 & 0.21369011 & 0.30218272 & 0.049112857 & 0.10540303 & 0.21009511 & 0.364611 \\
\hline 175 & phthalimide & 0.022862488 & 0.11137942 & 0.25970175 & 0.39394366 & 0.070836498 & 0.15258229 & 0.26158882 & 0.485008 \\
\hline 176 & piperidine & 0.0374539 & 0.26105743 & 0.56387769 & 0.86238902 & 0.16666511 & 0.35175487 & 0.57807063 & 1.096491 \\
\hline 177 & polyacrylamide & 0.01387697 & 0.079889281 & 0.19977215 & 0.2935384 & 0.053074367 & 0.11274366 & 0.19639667 & 0.362215 \\
\hline 178 & propanal & 0.01614299 & 0.14745202 & 0.21471382 & 0.37830883 & 0.080967787 & 0.17791732 & 0.24987472 & 0.50876 \\
\hline
\end{tabular}




\begin{tabular}{|c|c|c|c|c|c|c|c|c|c|}
\hline 179 & propionic acid & 0.008889695 & 0.071775649 & 0.15453033 & 0.23519568 & 0.039171452 & 0.084937919 & 0.16173523 & 0.285845 \\
\hline 180 & propyl amine & 0.031055852 & 0.23893691 & 0.31703291 & 0.58702567 & 0.1314777 & 0.2848379 & 0.3697666 & 0.786082 \\
\hline 181 & propylene & 0.001435262 & 0.020265144 & 0.21166796 & 0.23336837 & 0.025220231 & 0.04858231 & 0.19015683 & 0.263959 \\
\hline 182 & propylene glycol & 0.021734028 & 0.20017088 & 0.19470171 & 0.41660662 & 0.0860673 & 0.20969526 & 0.24311702 & 0.53888 \\
\hline 183 & propylene oxide & 0.022569162 & 0.21432978 & 0.22753489 & 0.46443383 & 0.093924478 & 0.22872451 & 0.27897739 & 0.601626 \\
\hline 184 & pyrazole & 0.083048922 & 0.75724402 & 0.48635045 & 1.3266434 & 0.34149506 & 1.0204274 & 0.66797361 & 2.029896 \\
\hline 185 & styrene & 0.012719724 & 0.11439759 & 0.21857346 & 0.34569078 & 0.056166543 & 0.13045384 & 0.25380061 & 0.440421 \\
\hline 186 & tert-butyl amine & 0.036485013 & 0.27484248 & 0.39498755 & 0.70631504 & 0.14702223 & 0.33336102 & 0.4308084 & 0.911192 \\
\hline 187 & tetrachloroethylene & 0.00560625 & 0.09772966 & 0.072410905 & 0.17574682 & 0.068270691 & 0.1663544 & 0.078053697 & 0.312679 \\
\hline 188 & tetrafluoroethane & 0.052334521 & 0.39243117 & 0.23110356 & 0.67586925 & 0.13851513 & 0.36359083 & 0.33270932 & 0.834815 \\
\hline 189 & tetrahydrofuran & 0.030157956 & 0.24300501 & 0.24227654 & 0.51543951 & 0.13043164 & 0.28175101 & 0.31253247 & 0.724715 \\
\hline 190 & toluene & 0.003804438 & 0.022091792 & 0.19867592 & 0.22457215 & 0.027398818 & 0.052252267 & 0.17902216 & 0.258673 \\
\hline 191 & trichloroacetic acid & 0.021486248 & 0.19030671 & 0.11080036 & 0.32259332 & 0.075569622 & 0.18962848 & 0.16904166 & 0.43424 \\
\hline 192 & trichloroborane & 0.022765938 & 0.20043845 & 0.07435625 & 0.29756064 & 0.07129034 & 0.18857445 & 0.12466623 & 0.384531 \\
\hline 193 & trichloroethylene & 0.021401867 & 0.19209271 & 0.1122341 & 0.32572868 & 0.075907964 & 0.18736437 & 0.17181032 & 0.435083 \\
\hline 194 & trichloromethane & 0.008935893 & 0.13703905 & 0.071317881 & 0.21729282 & 0.065216512 & 0.26538276 & 0.092974588 & 0.423574 \\
\hline 195 & trichloropropane & 0.016593939 & 0.14556039 & 0.1181719 & 0.28032623 & 0.058403314 & 0.17883339 & 0.14471029 & 0.381947 \\
\hline 196 & triethyl amine & 0.012823233 & 0.082886841 & 0.24694288 & 0.34265295 & 0.0585564 & 0.11990666 & 0.24103559 & 0.419499 \\
\hline 197 & trifluoroacetic acid & 0.060105618 & 0.47402239 & 0.25362043 & 0.78774843 & 0.17377244 & 0.45008315 & 0.38662819 & 1.010484 \\
\hline 198 & trifluoromethane & 0.041824194 & 0.36109828 & 0.22083199 & 0.62375447 & 0.15065239 & 0.58443544 & 0.26927191 & 1.00436 \\
\hline 199 & trimesoyl chloride & 0.036184854 & 0.34226486 & 0.34644564 & 0.72489536 & 0.15432539 & 0.43075015 & 0.38325723 & 0.968333 \\
\hline 200 & trimethyl borate & 0.016337145 & 0.12095114 & 0.17644513 & 0.31373342 & 0.048802913 & 0.12170234 & 0.18067322 & 0.351178 \\
\hline 201 & trimethylamine & 0.01078338 & 0.070356744 & 0.23720373 & 0.31834385 & 0.044666584 & 0.091991403 & 0.22710339 & 0.363761 \\
\hline 202 & vinyl acetate & 0.011544709 & 0.0885283 & 0.17754119 & 0.2776142 & 0.046318131 & 0.10063863 & 0.18674896 & 0.333706 \\
\hline 203 & vinyl chloride & 0.003461592 & 0.023562049 & 0.12428536 & 0.151309 & 0.028157207 & 0.05604362 & 0.12051209 & 0.204713 \\
\hline 204 & vinyl fluoride & 0.03651662 & 0.28642524 & 0.13074348 & 0.45368534 & 0.15924983 & 0.35027057 & 0.20513363 & 0.714654 \\
\hline 205 & xylene & 0.010307251 & 0.024795629 & 0.20418598 & 0.23928886 & 0.029845243 & 0.057661311 & 0.1846457 & 0.272152 \\
\hline 206 & octabenzone & 0.024431671 & 0.1756771 & 0.25413773 & 0.4542465 & 0.10172699 & 0.2207008 & 0.28696886 & 0.609397 \\
\hline 207 & succinic acid & 0.012991552 & 0.10063638 & 0.17828979 & 0.29191772 & 0.063871316 & 0.13009463 & 0.19691204 & 0.390878 \\
\hline 208 & sulfamic acid & 0.0204945 & 0.11544706 & 0.10106143 & 0.23700299 & 0.043384606 & 0.11286483 & 0.11227479 & 0.268524 \\
\hline 209 & tetrafluoroethylene & 0.063284253 & 1.1628227 & 0.34299943 & 1.5691064 & 2.4382847 & 4.5163659 & 0.4321636 & 7.386814 \\
\hline
\end{tabular}




\begin{tabular}{|c|c|c|c|c|c|c|c|c|c|}
\hline 210 & 1,1-dimethylcyclopentane & 0.008518378 & 0.041320208 & 0.21583234 & 0.26567092 & 0.026183073 & 0.047767963 & 0.20139837 & 0.275349 \\
\hline 211 & 1-pentanol & 0.018500141 & 0.14700442 & 0.25468072 & 0.42018528 & 0.10350623 & 0.20730051 & 0.28937836 & 0.600185 \\
\hline 212 & 2,3-dimethylbutan & 0.008518378 & 0.041320208 & 0.21583234 & 0.26567092 & 0.026183073 & 0.047767963 & 0.20139837 & 0.275349 \\
\hline 213 & 2-methylpentane & 0.008518378 & 0.041320208 & 0.21583234 & 0.26567092 & 0.026183073 & 0.047767963 & 0.20139837 & 0.275349 \\
\hline 214 & 3-methyl-1-butanol & 0.018500141 & 0.14700442 & 0.25468072 & 0.42018528 & 0.10350623 & 0.20730051 & 0.28937836 & 0.600185 \\
\hline 215 & acetonitrile & 0.017555497 & 0.093891905 & 0.33285856 & 0.44430596 & 0.074230888 & 0.15298892 & 0.3122633 & 0.539483 \\
\hline 216 & acrylonitrile & 0.013107189 & 0.069859809 & 0.24608641 & 0.3290534 & 0.055044786 & 0.11348897 & 0.2309276 & 0.399461 \\
\hline 217 & boron trifluoride & 0.042013194 & 0.26388822 & 0.13530163 & 0.44120304 & 0.059026739 & 0.20782464 & 0.16658752 & 0.433439 \\
\hline 218 & isoproturon & 0.036752712 & 0.27137106 & 0.28351917 & 0.59164293 & 0.13899085 & 0.31899921 & 0.35392095 & 0.811911 \\
\hline 219 & mecoprop & 0.027672876 & 0.22367478 & 0.22561346 & 0.47696112 & 0.10248073 & 0.23845039 & 0.28982971 & 0.630761 \\
\hline 220 & methylcyclopentane & 0.008518378 & 0.041320208 & 0.21583234 & 0.26567092 & 0.026183073 & 0.047767963 & 0.20139837 & 0.275349 \\
\hline 221 & napropamide & 0.085850618 & 0.96104953 & 0.40830648 & 1.4552066 & 0.24888582 & 2.1640092 & 0.49999872 & 2.912894 \\
\hline 222 & propane & 0.005011208 & 0.024915823 & 0.16191427 & 0.1918413 & 0.019749252 & 0.035532149 & 0.14780694 & 0.203088 \\
\hline 223 & pyridine & 0.03378544 & 0.23554895 & 0.54314276 & 0.81247715 & 0.15302354 & 0.32211498 & 0.55079234 & 1.025931 \\
\hline 224 & triethanolamine & 0.010917699 & 0.11329048 & 0.20332349 & 0.32753167 & 0.053633774 & 0.1149142 & 0.20764013 & 0.376188 \\
\hline
\end{tabular}


Table S5 Optimized hyper-parameters of deep learning neural network for LCA in eight impact categories

\begin{tabular}{|c|c|c|c|c|c|c|c|c|}
\hline & $\begin{array}{c}\text { EI99 - } \\
\text { Ecosystem }\end{array}$ & $\begin{array}{l}\text { EI99 - } \\
\text { Human } \\
\text { health }\end{array}$ & $\begin{array}{c}\text { EI99 - } \\
\text { Resources }\end{array}$ & $\begin{array}{l}\text { EI99 - } \\
\text { Total }\end{array}$ & $\begin{array}{c}\text { ReCiPe - } \\
\text { Ecosystem }\end{array}$ & $\begin{array}{c}\text { ReCiPe - } \\
\text { Human } \\
\text { health }\end{array}$ & $\begin{array}{l}\text { ReCiPe - } \\
\text { Resources }\end{array}$ & $\begin{array}{l}\text { ReCiPe - } \\
\text { Total }\end{array}$ \\
\hline $\begin{array}{c}\text { Number of hidden } \\
\text { layers }\end{array}$ & 2 & 3 & 3 & 2 & 3 & 3 & 3 & 3 \\
\hline $\begin{array}{l}\text { Number of neurons } \\
\text { in each hidden layer }\end{array}$ & 512 & 512 & 128 & 64 & 128 & 64 & 512 & 512 \\
\hline Activation Function & ReLu & ReLu & Sigmoid & ReLu & ReLu & ReLu & Sigmoid & ReLu \\
\hline
\end{tabular}


Table S6 Thirty most similar chemicals based on Euclidean distance

\begin{tabular}{|c|c|c|}
\hline & The SMILE structure of similar chemicals & $\begin{array}{l}\text { Euclidean distance } \\
\text { after PCA }\end{array}$ \\
\hline 0 & $\mathrm{O}=\mathrm{C}(\mathrm{OC}(=\mathrm{O}) \mathrm{C}(\mathrm{F})(\mathrm{F}) \mathrm{F}) \mathrm{C}(\mathrm{F})(\mathrm{F}) \mathrm{F}$ & 0.000 \\
\hline 1 & $\mathrm{O}=\mathrm{C}(\mathrm{OCC}(\mathrm{F})(\mathrm{F}) \mathrm{F}) \mathrm{C}(\mathrm{F})(\mathrm{F}) \mathrm{F}$ & 2.534 \\
\hline 2 & $\mathrm{O}=\mathrm{C}(\mathrm{NOC}(=\mathrm{O}) \mathrm{C}(\mathrm{F})(\mathrm{F}) \mathrm{F}) \mathrm{C}(\mathrm{F})(\mathrm{F}) \mathrm{F}$ & 2.847 \\
\hline 3 & $\mathrm{C}=\mathrm{CCOC}(=\mathrm{O}) \mathrm{C}(\mathrm{F})(\mathrm{F}) \mathrm{C}(\mathrm{F})(\mathrm{F}) \mathrm{F}$ & 2.949 \\
\hline 4 & $\mathrm{C}=\mathrm{C}(\mathrm{F}) \mathrm{C}(=\mathrm{O}) \mathrm{OC}(\mathrm{F})(\mathrm{F}) \mathrm{C}(\mathrm{C})(\mathrm{F}) \mathrm{F}$ & 3.062 \\
\hline 5 & $\mathrm{C}=\mathrm{COC}(=\mathrm{O}) \mathrm{C}(\mathrm{F})(\mathrm{F}) \mathrm{C}(\mathrm{F})(\mathrm{F}) \mathrm{C}(\mathrm{F})(\mathrm{F}) \mathrm{F}$ & 3.063 \\
\hline 6 & $\operatorname{CCOC}(=\mathrm{O}) \mathrm{C}(\mathrm{F})(\mathrm{F}) \mathrm{C}(\mathrm{F})(\mathrm{F}) \mathrm{F}$ & 3.121 \\
\hline 7 & $\operatorname{CCOC}(=\mathrm{O}) \mathrm{C}=\mathrm{C}(\mathrm{C}(\mathrm{F})(\mathrm{F}) \mathrm{F}) \mathrm{C}(\mathrm{F})(\mathrm{F}) \mathrm{F}$ & 3.155 \\
\hline 8 & $\mathrm{C}=\mathrm{CC}(=\mathrm{O}) \mathrm{OCC}(\mathrm{F})(\mathrm{F}) \mathrm{C}(\mathrm{F})(\mathrm{F}) \mathrm{F}$ & 3.167 \\
\hline 9 & $\mathrm{COC}(=\mathrm{O}) \mathrm{C}(\mathrm{F})(\mathrm{F}) \mathrm{C}(\mathrm{F})(\mathrm{F}) \mathrm{C}(\mathrm{F})(\mathrm{F}) \mathrm{F}$ & 3.189 \\
\hline 10 & $\mathrm{COC}(=\mathrm{O}) \mathrm{C}(\mathrm{F})(\mathrm{F}) \mathrm{C}(\mathrm{F})=\mathrm{C}(\mathrm{F}) \mathrm{F}$ & 3.292 \\
\hline 11 & $\mathrm{O}=\mathrm{C}([\mathrm{O}-]) \mathrm{C}(\mathrm{F})(\mathrm{F}) \mathrm{C}(\mathrm{F})(\mathrm{F}) \mathrm{C}(\mathrm{F}) \mathrm{F}$ & 3.303 \\
\hline 12 & $\mathrm{COC}(=\mathrm{O}) \mathrm{C}(\mathrm{F})(\mathrm{F}) \mathrm{C}(\mathrm{F})(\mathrm{F}) \mathrm{F}$ & 3.323 \\
\hline 13 & $\mathrm{C}=\mathrm{CC}(=\mathrm{O}) \mathrm{OC}(\mathrm{C}(\mathrm{F})(\mathrm{F}) \mathrm{F}) \mathrm{C}(\mathrm{F})(\mathrm{F}) \mathrm{F}$ & 3.325 \\
\hline 14 & $\mathrm{C}=\mathrm{C}(\mathrm{C}) \mathrm{C}(=\mathrm{O}) \mathrm{OCC}(\mathrm{F})(\mathrm{F}) \mathrm{C}(\mathrm{F})(\mathrm{F}) \mathrm{F}$ & 3.328 \\
\hline 15 & $\operatorname{CCCOC}(=\mathrm{O}) \mathrm{C}(\mathrm{F})(\mathrm{F}) \mathrm{C}(\mathrm{F})(\mathrm{F}) \mathrm{F}$ & 3.370 \\
\hline 16 & $\operatorname{CCOC}(=\mathrm{O}) \mathrm{C}(\mathrm{F})(\mathrm{F}) \mathrm{C}(\mathrm{F})(\mathrm{F}) \mathrm{C}(\mathrm{F})(\mathrm{F}) \mathrm{F}$ & 3.473 \\
\hline 17 & $\mathrm{C}=\mathrm{CCOC}(=\mathrm{O}) \mathrm{C}(\mathrm{F})(\mathrm{F}) \mathrm{C}(\mathrm{F})(\mathrm{F}) \mathrm{C}(\mathrm{F})(\mathrm{F}) \mathrm{F}$ & 3.498 \\
\hline 18 & $\mathrm{O}=\mathrm{C}(\mathrm{O}) \mathrm{C}(\mathrm{F})(\mathrm{F}) \mathrm{C}(\mathrm{F})(\mathrm{F}) \mathrm{C}(\mathrm{F})(\mathrm{F}) \mathrm{F}$ & 3.500 \\
\hline 19 & $\mathrm{C}=\mathrm{CC}(=\mathrm{O}) \mathrm{OCC}(\mathrm{F})(\mathrm{F}) \mathrm{C}(\mathrm{F})(\mathrm{F}) \mathrm{C}(\mathrm{F})(\mathrm{F}) \mathrm{F}$ & 3.599 \\
\hline 20 & $\mathrm{O}=\mathrm{C}(\mathrm{O}) \mathrm{C}(\mathrm{F})(\mathrm{F}) \mathrm{C}(\mathrm{F})(\mathrm{F}) \mathrm{C}(\mathrm{F}) \mathrm{F}$ & 3.627 \\
\hline 21 & $\mathrm{O}=\mathrm{C}(\mathrm{C}=\mathrm{C}(\mathrm{O}) \mathrm{C}(\mathrm{F})(\mathrm{F}) \mathrm{F}) \mathrm{C}(\mathrm{F})(\mathrm{F}) \mathrm{F}$ & 3.632 \\
\hline
\end{tabular}


$22 \operatorname{COC}(=\mathrm{O}) \mathrm{CC}(=\mathrm{O}) \mathrm{C}(\mathrm{F})(\mathrm{F}) \mathrm{C}(\mathrm{F})(\mathrm{F}) \mathrm{F}$

$23 \mathrm{O}=\mathrm{C}(\mathrm{Cl}) \mathrm{ON}(\mathrm{C}(\mathrm{F})(\mathrm{F}) \mathrm{F}) \mathrm{C}(\mathrm{F})(\mathrm{F}) \mathrm{F}$

$24 \mathrm{C}=\mathrm{CC}(=\mathrm{O}) \mathrm{OCCC}(\mathrm{F})(\mathrm{F}) \mathrm{C}(\mathrm{F})(\mathrm{F}) \mathrm{F}$

$25 \mathrm{O}=\mathrm{C}(\mathrm{CC}(=\mathrm{O}) \mathrm{C}(\mathrm{F})(\mathrm{F}) \mathrm{F}) \mathrm{C}(\mathrm{F})(\mathrm{F}) \mathrm{F}$

$26 \mathrm{C}=\mathrm{C}(\mathrm{C}) \mathrm{C}(=\mathrm{O}) \mathrm{OC}(\mathrm{C}(\mathrm{F})(\mathrm{F}) \mathrm{F}) \mathrm{C}(\mathrm{F})(\mathrm{F}) \mathrm{F}$ 3.744

$27 \mathrm{C}=\mathrm{C}(\mathrm{C}) \mathrm{C}(=\mathrm{O}) \mathrm{OCC}(\mathrm{F})(\mathrm{F}) \mathrm{C}(\mathrm{F}) \mathrm{C}(\mathrm{F})(\mathrm{F}) \mathrm{F}$

3.770

$28 \mathrm{CC}(=\mathrm{CC}(=\mathrm{O}) \mathrm{C}(\mathrm{F})(\mathrm{F}) \mathrm{F}) \mathrm{C}(\mathrm{F})(\mathrm{F}) \mathrm{F}$

3.806

$29 \mathrm{O}=\mathrm{C}(\mathrm{OCCOC}(=\mathrm{O}) \mathrm{C}(\mathrm{F})(\mathrm{F}) \mathrm{F}) \mathrm{C}(\mathrm{F})(\mathrm{F}) \mathrm{F}$

3.809 


\section{References}

(1) Lauria, A.; Ippolito, M.; Almerico, A. M., Brief communication: Principal component analysis on molecular descriptors as an alternative point of view in the search of new Hsp90 inhibitors. Comput. Biol. Chem. 2009, 33 (5), 386-390.

https://doi.org/10.1016/j.compbiolchem.2009.07.010

(2) Ho, C.; Yi, J.; Wang, X., Biocatalytic Continuous Manufacturing of Diabetes Drug: Plantwide Process Modeling, Optimization, and Environmental and Economic Analysis. ACS Sustain. Chem. Eng. 2019, 7 (1), 1038-1051. https://doi.org/10.1021/acssuschemeng.8b04673 (3) Ott, D.; Borukhova, S.; Hessel, V., Life cycle assessment of multi-step rufinamide synthesis-from isolated reactions in batch to continuous microreactor networks. Green Chem. 2016, 18 (4), 1096-1116. https://doi.org/10.1039/C5GC01932J

(4) Kim, S.; Overcash, M. R. J. J. o. C. T.; Biotechnology, Energy in chemical manufacturing processes: gate-to-gate information for life cycle assessment. 2003, 78 (9), 995-1005.

https://doi.org/10.1002/jctb.821 